\title{
Properties of nuclei in the nobelium region studied within the covariant, Skyrme, and Gogny energy density functionals
}

J. Dobaczewski ${ }^{1-4}$, A.V. Afanasjev ${ }^{5}$, M. Bender ${ }^{6,7}$, L.M. Robledo ${ }^{8}$, and Yue $\mathrm{Shi}^{9}$ (石跃)

${ }^{1}$ Department of Physics, University of York, Heslington, York YO10 5DD, United Kingdom

${ }^{2}$ Department of Physics, P.O. Box 35 (YFL), University of Jyväskylä FI-40014 Jyväskylä, Finland

${ }^{3}$ Institute of Theoretical Physics, Faculty of Physics, University of Warsaw Pasteura 5, PL-02-093 Warsaw, Poland

${ }^{4}$ Helsinki Institute of Physics, P.O. Box 64, FI-00014 Helsinki, Finland

${ }^{5}$ Department of Physics and Astronomy, Mississippi State University Mississippi State, Mississippi 39762, USA

${ }^{6}$ Université de Bordeaux, Centre d'Etudes Nucléaires de Bordeaux Gradignan UMR5797, F-33175 Gradignan, France

${ }^{7}$ CNRS/IN2P3, Centre d'Etudes Nucléaires de Bordeaux Gradignan UMR5797, F-33175 Gradignan, France

${ }^{8}$ Departamento de Física Teórica, Universidad Autónoma de Madrid E-28049 Madrid, Spain

${ }^{9}$ National Superconducting Cyclotron Laboratory, Michigan State University, East Lansing, Michigan, 48824-1321, USA

\section{Abstract}

We calculate properties of the ground and excited states of nuclei in the nobelium region for proton and neutron numbers of $92 \leq Z \leq 104$ and $144 \leq N \leq 156$, respectively. We use three different energy-densityfunctional (EDF) approaches, based on covariant, Skyrme, and Gogny functionals, each with two different parameter sets. A comparative analysis of the results obtained for quasiparticle spectra, odd-even and two-particle mass staggering, and moments of inertia allows us to identify single-particle and shell effects that are characteristic to these different models and to illustrate possible systematic uncertainties related to using the EDF modelling.

Keywords: heavy and superheavy nuclei; nuclear masses; quasiparticle 
excitations; odd-even mass staggering; two-particle mass staggering; moments of inertia; nuclear energy density functionals 


\section{Introduction}

Recent experimental studies of nuclei in the nobelium region provided rich spectroscopic data $[1,2]$, which, in principle, can be used as a benchmark information for extrapolations into the region of superheavy nuclei. Numerous theoretical studies are aimed at modelling of these spectroscopic data $[3,4,5,6,7,8,9,10,11,12,13,14,15,16,17,18,19,20,21]$ so as to make such extrapolations as reliable as possible.

The estimation of theoretical uncertainties is one of the most essential aspects of extrapolating nuclear models into exotic nuclei [22]. One, fairly easy part of it, is the evaluation of statistical uncertainties of observables that are related to the uncertainties of model parameters adjusted, in one way or another, to experimental data. Another one, very difficult, pertains to those systematic uncertainties related to the definition and contents of the different terms that make up the models themselves. An obvious strategy, which, anyhow, gives us only a limited glimpse on possible systematic uncertainties, is to study a set of variants of a given model, and to analyze differences obtained for calculated observables.

In the present study, we aim at such an analysis of results obtained within three fairly different energy-density-functional (EDF) approaches. Namely, we employ the covariant EDFs [9], with one classic (NL1 [23]) and one recent (NL3* [24]) parameter set, Skyrme EDFs [25], with one classic (SLy4 [26]) and one recent (UNEDF2 [27]) parameter set, as well as Gogny EDFs [28], again with one classic (D1S [29]) and one recent (D1M [30]) parameter set.

Our goal is thus to determine, present, and compare results obtained within these six models for a common set of calculated observables. We aim at performing these analyses within the most similar and/or equivalent conditions, so as to meaningfully discuss general qualitative similarities and differences. In all cases, pairing correlations are treated on the Bogoliubov level. In the literature, it is customary to label such calculations as Hartree-Fock-Bogoliubov (HFB) in the non-relativistic cases and as relativistic Hartree-Bogoliubov (RHB) for the specific variant of relativistic mean-field model used here, thereby emphasizing that HFB-like equations are solved instead of simpler HF+BCS equations. Only in the calculations with a Gogny force, however, the same effective interaction is used to determine direct, exchange, and pairing matrix elements. By contrast, in case of Skyrme EDF and the relativistic approach particle-hole and pairing matrix elements relate to different effective interactions, which can be used to 
simplify their form and phenomenological adjustment. Also, in the RHB approach all exchange terms are neglected, whereas in the case of many Skyrme parameterizations some are modified in order to improve the description of data [25]. None of these formal differences is relevant for our discussion, and we will use whenever possible the generic notion of an EDF method for all three approaches. Indeed, in all cases the total energy can be cast into the form of a functional of normal and anomalous one-body density matrices from which the equations-of-motion are then derived by variation. We do not attribute too much of an importance to quantitative similarities and differences between the obtained results, especially when the models are compared to experimental data. Indeed, a detailed agreement with the data may crucially depend on specific model-parameter adjustments, or on various corrections taken into account or disregarded. The phenomenological EDF used include a limited set of parameters (typically between seven and twenty) and aim at a global description of a wide variety of nuclear properties and therefore a perfect agreement with experimental data is out of reach at present. Certainly, in the future all EDF approaches will be improved; here we only look into generic properties obtained for selected current global parameterisations thereof.

In the present analysis, we systematically calculated the ground states of even-even and odd-mass nuclei from uranium $(Z=92)$ to rutherfordium $(Z=104)$ and for neutron numbers between $N=144$ and 156. The selection of this region of heaviest actinides/lightest superheavy nuclei is guided by the need for reliable experimental data on spectroscopic properties (in particular, on the single-particle energies of deformed one-quasiparticle states) based on which the extrapolability of a given theory/functional towards region of superheavy nuclei may be judged. In addition, we determined low-lying quasiparticle spectra of odd-mass nuclei and low-spin moments of inertia of even-even nuclei. The main thrust of the analysis was on the attempt to identify single-particle and shell-structure properties of these nuclei by looking at many-body observables such as masses, odd-even and two-particle mass staggering, and excitation energies.

The paper is organised as follows. Selected theoretical aspects of our calculations are presented in section 2, with four subsections discussing the methods related to obtaining results for the Skyrme EDF SLy4 (2.1), Skyrme EDF UNEDF2 (2.2), Gogny EDFs (2.3), and covariant EDFs (2.4). The results of the calculations are given in section 3, with subsections devoted to the Nilsson diagrams (3.1), quasiparticle spectra (3.2 and 3.3), odd-even and 
two-particle mass staggering (3.4), and moments of inertia (3.5). Conclusions are presented in section 4 .

\section{Calculation details}

\subsection{Skyrme energy density functional SLy4}

For the Skyrme EDF SLy4 [26], the calculations were carried out with the Skyrme HFB solver CR8 whose development over the years has been documented in Refs. [31, 32, 33, 34]. It uses a 3D coordinate space mesh representation of single-particle states along the lines of the solver EV8 described in Ref. [35], but is extended in such a way that intrinsic timereversal invariance can be broken and that HFB equations are solved instead of $\mathrm{HF}+\mathrm{BCS}$. Single-particle states are represented in a cubic box of $32^{3} \mathrm{fm}^{3}$ with a step size of $0.8 \mathrm{fm}$ between discretization points. Imposing triaxial symmetry, only $1 / 8$ of the box has to be represented numerically, meaning that only a $20 \times 20 \times 20$ mesh is to be treated.

When calculated with SLy4, the ground states of even-even nuclei considered here are all axial, and the blocked states of odd- $A$ nuclei also remain almost axial. All blocked calculations were initialized with the ground states of adjacent even-even nuclei. Self-consistent blocking was performed by considering the quasiparticle state dominated by a given eigenstate of the single-particle Hamiltonian and by exchanging the corresponding columns of the HFB $U$ and $V$ matrices after the diagonalization of the HFB Hamiltonian, which in turn was constructed using the mean fields of the blocked solution from the previous iteration, see, e.g., Refs. [32, 36, 37].

To avoid mixing of quasiparticle states with different average values of the angular momentum component $\left\langle\hat{j}_{\|}\right\rangle$parallel to the symmetry axis of the initial configuration in the diagonalization of the HFB Hamiltonian, the many-body expectation value of $\left\langle\hat{J}_{\|}\right\rangle$was held fixed with a cranking constraint at the value of $\left\langle\hat{J}_{\|}\right\rangle$equal to the one of the blocked quasiparticle state. As the code CR8 allows for triaxial shapes the mixing cannot be fully suppressed. As a consequence, the blocked HFB states are not necessarily orthogonal even when they have different average value of $\left\langle\hat{J}_{\|}\right\rangle$.

In this respect, blocking the $\left\langle\hat{j}_{\|}\right\rangle=1 / 2$ levels presents a particular difficulty. Without a cranking constraint, the code CR8 very often converges

toward a solution where the many-body expectation value $\left\langle\hat{J}_{\|}\right\rangle$is close to zero, and where the blocked quasiparticle in the spectrum of eigenstates of 
the HFB Hamiltonian is mixed with other low-lying quasiparticles of different $\left\langle\hat{j}_{\|}\right\rangle$. For these states, using or not using the cranking constraint might in some cases make a difference of the order of 100 to $200 \mathrm{keV}$. An example is the ground state of ${ }^{251} \mathrm{Cf}$. In blocked calculations with cranking constraint, there is a low lying $\left\langle\hat{J}_{\|}\right\rangle^{\pi}=1 / 2^{+}$level at $45 \mathrm{keV}$ excitation energy above the calculated $\left\langle\hat{J}_{\|}\right\rangle^{\pi}=3 / 2^{+}$ground state. In the calculations without cranking constraint, the energy of the $3 / 2^{+}$state does not change much, but the $1 / 2^{+}$ level is lowered by about $180 \mathrm{keV}$ and becomes the ground state, in agreement with experiment, but at the expense of the blocked quasiparticle being a strong mixture of $\left\langle\hat{j}_{\|}\right\rangle=1 / 2$ and $\left\langle\hat{j}_{\|}\right\rangle=3 / 2$, and of having an angular momentum $\left\langle\hat{J}_{\|}\right\rangle$that cannot be easily interpreted within the strong coupling model anymore. For blocked states with higher $\left\langle\hat{j}_{\|}\right\rangle$, the mixing is always much smaller.

For the coupling constants of the so-called time-odd terms that contribute to cranked and blocked states, the same "hybrid" choice was made as in many earlier calculations [7, 31, 32, 33, 38, 39, 40, 41, 42, 43, 44]. They were set to the "native" values dictated by a density-dependent Skyrme twobody force for all terms except for those that multiply terms that couple two derivatives and two Pauli spin matrices. The latter were set to zero for reasons of Galilean invariance and internal consistency, cf. Refs. [31, 38] for further discussion.

In the present study, neutron and proton surface pairing interactions were used, with the strengths adjusted to the three-point gaps $\Delta_{n}^{(3)}$ of ${ }^{247} \mathrm{Cf}(N=$ $149)$ and $\Delta_{p}^{(3)}$ of ${ }^{249} \mathrm{Bk}(Z=97)$, as defined by Eqns. (7) and (6) below, leading to

$$
\begin{aligned}
& V_{n}=-1240 \mathrm{MeV} \mathrm{fm}^{3} \\
& V_{p}=-1575 \mathrm{MeV} \mathrm{fm}^{3}
\end{aligned}
$$

The pairing-active spaces were limited by the soft cutoffs of $5 \mathrm{MeV}$ above and below the neutron and proton Fermi energies as described in Ref. [45]. With that, the proton pairing strength is significantly larger than that given by the standard value of $V_{n}=V_{p}=-1250 \mathrm{MeV} \mathrm{fm}^{3}$ used for heavy nuclei in previous publications [39, 7, 40, 41, 42, 43] (which all have been carried out using the Lipkin-Nogami (LN) scheme, though). These previous values were obtained by adjusting moments of inertia in rotational bands of heavy nuclei [45]. There is, however, also some published work on very heavy nuclei $[44,46]$ where the pairing interaction of volume type was adjusted to some 
$\Delta_{q}^{(3)}$ values of odd- $A$ actinide nuclei.

In principle, the like-particle pairing interaction should be of isovector type, which implies $V_{n}=V_{p}$. Differences between the adjusted neutron and proton pairing strengths can have many reasons: (i) the compensation of imperfections of the calculated single-particle spectra, (ii) the compensations of the cutoff energy that is chosen to be the same in neutron and proton phase spaces (iii) the compensation of the imperfections of the chosen form of the pairing interaction itself. Note that if there were none of the above mentioned deficiencies, the proton pairing strength would have to be smaller than the neutron pairing strength, so as to compensate for the absence of Coulomb pairing in our calculations.

Our attempts to adjust the neutron pairing strength to $\Delta_{n}^{(3)}$ in ${ }^{251} \mathrm{Cf}$ $(N=153)$, as done in Ref. [21] and in the present work for UNEDF2, led to the values of $V_{n}=-1060$ and $V_{p}=-1565 \mathrm{MeV} \mathrm{fm}^{3}$. With such much weaker neutron pairing strength, in ground states of many of the lighter odd- $N$ nuclei, the pairing disappeared. This seems to be connected to an anomaly of the calculated single-particle spectra of heavy $N=153$ isotones that translates into a much larger values of $\Delta_{n}^{(3)}$ for these nuclei than for the neighbouring ones.

\subsection{Skyrme energy density functional UNEDF2}

For the Skyrme EDF UNEDF2 [27], the calculations were performed using the symmetry-unrestricted code HFODD (v268h) [47]. The HFB equations were solved by expanding single-particle wave functions on 680 deformed harmonic-oscillator ( $\mathrm{HO}$ ) basis states, with $\mathrm{HO}$ frequencies of $\hbar \Omega_{x}=\hbar \Omega_{y}=$ $8.4826549 \mathrm{MeV}$ and $\hbar \Omega_{z}=6.4653456 \mathrm{MeV}$. This corresponds to including the HO basis states up to $N_{x}=N_{y}=13$ and $N_{z}=14$.

The time-odd coupling constants of the Skyrme EDF UNEDF2 were determined by the local-gauge-invariance arguments, as defined in Ref. [48]. The HFB equation adopted a quasiparticle cutoff energy of $60 \mathrm{MeV}$. Within this model space, the neutron and proton pairing strengths were readjusted to match the experimental values of $\Delta_{n}^{(3)}$ and $\Delta_{p}^{(3)}$ in ${ }^{251} \mathrm{Cf}$ and ${ }^{249} \mathrm{Bk}$, respectively. This resulted in values of

$$
\begin{aligned}
& V_{n}=-233.889 \mathrm{MeV} \mathrm{fm}^{3}, \\
& V_{p}=-280.330 \mathrm{MeV} \mathrm{fm}^{3}
\end{aligned}
$$

that are larger by about 25 and $50 \mathrm{MeV} \mathrm{fm}^{3}$, respectively, than those corresponding to the original UNEDF2 values, where the LN pairing corrections 
were included, though.

\subsection{Gogny energy density functionals}

Odd mass nuclei were described using the HFB method with full blocking and taking into account all possible time-odd fields coming from the potential part of the Gogny interaction. The original formulation of the densitydependent part of the interaction is preserved and therefore no additional time-odd density-dependent terms are added to it. This assumption has to be verified in the future as there are indications that such kind of terms could be required to recover basic properties of odd-odd systems [49].

The computer code ATB was used in the calculations [50]. Axial symmetry was preserved in the calculations but reflection symmetry was not. Therefore, the projection of the angular momentum along the intrinsic axial-symmetry axis, $\Omega=\left\langle\hat{j}_{\|}\right\rangle$, is a good quantum number, but this is not necessarily the case for parity. However, many of the states analyzed show a mean value of the parity operator, which is rather close to either plus or minus one, allowing the labelling of those states with a definite parity. As $\Omega$ is a good quantum number, states with different values of $\Omega$ are automatically orthogonal. States with the same $\Omega$ value, same parity and similar deformation parameters are imposed to be orthogonal to each other by using the traditional technique of constraints. The way the constraints are handled does not depend on the nature of the constrained operator as only its mean value and gradient are required [51]. The code expands the quasiparticle operators in a harmonic oscillator basis with 15 shells $(680 \times 2$ states $)$ although axial symmetry reduces the maximum size of matrices to $120 \times 2$, corresponding to the $\Omega=1 / 2$ block.

In the odd-mass (odd number parity) case, the HFB iterative minimization process requires a starting mean-field state with given characteristics. An HFB mean-field state with even number parity (obtained with a time-even code) was used to generate this starting configuration. The mean values of neutron $N$ and proton $Z$ number operators were constrained to the required values for the odd nucleus under consideration. To avoid spurious good-parity solutions, which could result from the propagation of self-consistent symmetries in the HFB method, a small octupole moment was also constrained.

Ten quasiparticle states with the lowest one-quasiparticle energies coming out of this calculation were considered as starting configurations for the timeodd code ATB. They were obtained with the standard "blocking procedure" consisting of swapping the appropriate columns in the $U$ and $V$ matrices of the Bogoliubov transformation. Each of the starting configurations was 
labelled with the corresponding $\Omega$ quantum number, which was preserved all along the minimization process. The first starting configuration with a given value of $\Omega$ usually converges to the lowest-energy band head with angular momentum $I=\Omega$. For the same value of $\Omega$, the second starting configuration usually converges to the same first solution, unless an orthogonality constraint to that state is imposed (including both the $\Omega$ and $-\Omega$ states). For the third starting configuration for a given $\Omega$ value, the orthogonality constraints with respect to the first and second states are required. In principle, for the $n$-th starting configuration $2(n-1)$ orthogonality constraints to the states previously obtained are required. Although the number of constraints can be relatively high, this is not a formal problem for the gradient method used in the code, as handling of constraints is very simple to implement [51]. The orthogonality constraint is also important to ensure the orthogonality of the physical states. If such orthogonality is not imposed, even if the states are different, the relative excitation energies can be shifted. In the present calculations only two starting configurations for each value of $\Omega$ were considered.

For the calculation of the properties of even-even nuclei required in the evaluation of the pairing gap parameters, the code HFBAXIAL was used. It has the capability to break reflection symmetry although the phenomenon was not relevant in the present calculation. Moments of inertia were determined using cranked HFB wave functions obtained with the code HFBTRI. In this code, the axial and time-reversal symmetries were allowed to be broken in the minimization process.

\subsection{Covariant energy density functionals}

The RHB equations $[52,8]$ were solved in the basis of an anisotropic threedimensional harmonic oscillator in Cartesian coordinates. For all nuclei and states determined in this work, the same basis deformation of $\beta_{0}=0.3$, $\gamma=0^{\circ}$ and oscillator frequency of $\hbar \omega_{0}=41 \mathrm{~A}^{-1 / 3} \mathrm{MeV}$ have been used. All fermionic and bosonic states belonging to the shells up to $N_{F}=14$ and $N_{B}=$ 20 were taken into account when performing diagonalization of the Dirac equation and matrix inversion of the Klein-Gordon equations, respectively. As follows from detailed analysis of Refs. [8, 13], this truncation of basis provides sufficient accuracy of the calculations.

As the effective interaction in the particle-particle $(p p)$ channel, the central part of the non-relativistic Gogny finite-range interaction was used. The clear advantage of such a pairing force is that it provides an automatic cutoff 
of high-momentum components. The motivation for such an approach to the description of pairing was given in Ref. [52]. The D1S parametrization of the Gogny force was used here. No specific adjustment of its strength was used, because it provided a reasonable description of the pairing indicators in ${ }^{249} \mathrm{Bk}$ and ${ }^{249,251} \mathrm{Cf}$ and moments of inertia in ${ }^{252,254}$ No [8].

Two different covariant EDFs, namely, NL1 [23] - fitted to the nuclei in the valley of beta-stability, and NL3* [24] - tailored towards the description of neutron-rich nuclei, were used in the current study. The covariant EDF NL1 was extensively used in the calculations of rotational bands across the nuclear chart (see Ref. [20]). Covariant EDF NL3* was less tested than NL1 with respect to the description of rotating nuclei. However, its global performance is well established [53]. Note that so far only these two covariant EDFs were systematically confronted with experimental data on single-particle states. For example, the study of predominantly single-particle states in odd-mass nuclei neighbouring to the doubly magic spherical nuclei was performed in Ref. [54] within the relativistic particle-vibration coupling model employing covariant EDF NL3*. In Ref. [13], the first systematic study of the singleparticle spectra in deformed nuclei in rare-earth region and actinides was performed with the covariant EDFs NL1 and NL3*.

It is interesting that the overall accuracy of the description of the energies of deformed one-quasiparticle states [13] is slightly better in the old covariant EDF NL1 than in the recent functional NL3*. This suggests that the inclusion of extra information on neutron rich nuclei into the fit of the functional NL3* may lead to some degradation of the description of single-particle states along the valley of beta-stability. Note that these two functionals well reproduce deformation properties of ground states of even-even actinides [8, 20] and indicate that they are axially symmetric.

A proper description of odd or rotating nuclei implies breaking of the time-reversal symmetry of the mean field, which is induced by the unpaired nucleon [55] or rotation [56]. As a consequence, time-odd mean fields and nucleonic currents, which cause the nuclear magnetism [57] have to be taken into account. In the covariant EDF, time-odd mean fields are defined through the Lorentz invariance, and thus they do not require additional coupling constants.

The effects of blocking due to the odd particle were included in a fully self-consistent way. This was done within the code CRHB, according to Refs. [58, 59, 60]. The blocked orbital was specified by different tags such as (i) dominant main oscillator quantum number $N$ of the wave function, (ii) 
dominant $\Omega$ quantum number of the wave function, (iii) particle or hole nature of the blocked orbital, and (iv) position of the state within the specific parity/signature/dominant- $N /$ dominant- $\Omega$. For a given odd-mass nucleus, possible blocked configurations were defined from the analysis of calculated quasiparticle spectra in neighboring even-even nuclei and the occupation probabilities of the single-particle orbitals of interest in these nuclei.

Note that in the cases when the calculations of odd-mass nuclei were performed only for the definition of the $\Delta^{(3)}$ indicators (see Sec. 3.4 below), we restricted the analysis to 5-6 one-quasiparticle configurations with expected lowest total energies, so as to properly determine the ground state of an odd-mass nucleus. The calculations confirmed the conclusion of the statistical analysis of Ref. [13] that absolute majority of the one-quasiparticle configurations are axially symmetric. However, some degree of triaxiality was obtained in the $\nu 3 / 2[622], \nu 1 / 2[501]$ and $\pi 1 / 2[400]$ configurations.

\section{Results}

In this section, we present the results obtained and we look for signatures of shell effects in the systematics of many-body observables. This is in addition to analyzing the eigenvalues of mean-field Hamiltonians as a function of deformation, that is, the Nilsson diagrams, which are not observables, but provide a useful illustration of the underlying single-particle structure.

We note here that the calculation of many-body observables implies a full self-consistency reached for every individual state, that is, for ground and excited states. For example, quasiparticle spectra, which we discuss below, always result from calculating differences of total energies, determined separately for different many-body self-consistent solutions. Because of that, each blocked HFB state may have a slightly different quadrupole, hexadecapole, or higher deformation (see, for example, the results of statistical analysis in Ref. [13]), which then feeds back to the mean and pairing fields. In this way, the deformation-polarization effects, exerted by one-quasiparticle states, are fully taken into account.

In odd-mass nuclei, quasiparticle excitations were obtained by blocking the relevant levels when performing the HFB calculations. The spectra were obtained by comparing the total energies of different configurations. The procedure closely followed that of Refs. [8, 37]. 


\subsection{Nilsson diagrams}

The Nilsson diagrams, shown in Figs. 1-3, have been obtained by diagonalizing the self-consistent mean-field Hamiltonians corresponding to the states that were constraint to a sequence of values of the axial mass quadrupole moment [60], $Q=\left\langle 2 z^{2}-x^{2}-y^{2}\right\rangle$. Then, by using a simple phenomenological formula [61],

$$
\beta_{2} \equiv \frac{4 \pi}{3 R^{2} A} \sqrt{\frac{5}{16 \pi}} Q \simeq Q \times 0.009 \mathrm{~b}^{-1},
$$

values of the average quadrupole moments were translated into values of the Bohr parameters $\beta_{2}$. The numerical factor of $0.009 \mathrm{~b}^{-1}$ corresponds to $R=1.2 \mathrm{fm} A^{1 / 3}$ and $A=254$. Note that the Nilsson diagrams obtained in this way constitute only an illustration of single-particle properties of the nuclei in the nobelium region. Indeed, going in either direction in the chart of nuclei, the relative distances between spherical and deformed levels change, closing some of the gaps visible in Figs. 1-3 while opening others. Examples for such evolution of the shell structure of spherical states and the deformed minima can be found in Refs. [43, 62, 63, 64]. Small, but clearly visible changes of the Nilsson diagrams can already be spotted when just adding a few neutrons or protons, compare for example the Nilsson diagram obtained with SLy4 for ${ }^{254}$ No, Fig. 1, with the one for ${ }^{250} \mathrm{Fm}$ presented in Refs [43], or the Nilsson diagrams obtained with NL1 and NL3* for ${ }^{254}$ No, Fig. 3, with those for ${ }^{244} \mathrm{Cm}$ presented in Ref. [20].

As we can see, for all considered EDFs, the overall positions and deformation-dependence of single-particle levels is fairly similar. In particular, deformed shell gaps, which appear near ground-state deformations of $\beta_{2}=$ 0.3 , occur at particle numbers of $Z=98, Z=104$ and $N=150$ and/or $N=152$ in the majority of the functionals. The only exception is the functional NL3*, which is characterized by additional gaps at $Z=102$ and $N=148$. Another deformed gap at $\beta_{2}=0.2$ is observed for $Z=110$. Moreover, significant differences in important details are also visible. The proton deformed shell gaps appear consistently above the one at $Z=100$ that is tentatively inferred from the experimental data, see discussion in Refs. [8, 21].

Interestingly, although different EDFs show similar deformed neutron shell closures, we observe dramatic differences in the shell structure at sphericity between the Skyrme EDF UNEDF2 and the other ones, as shown 

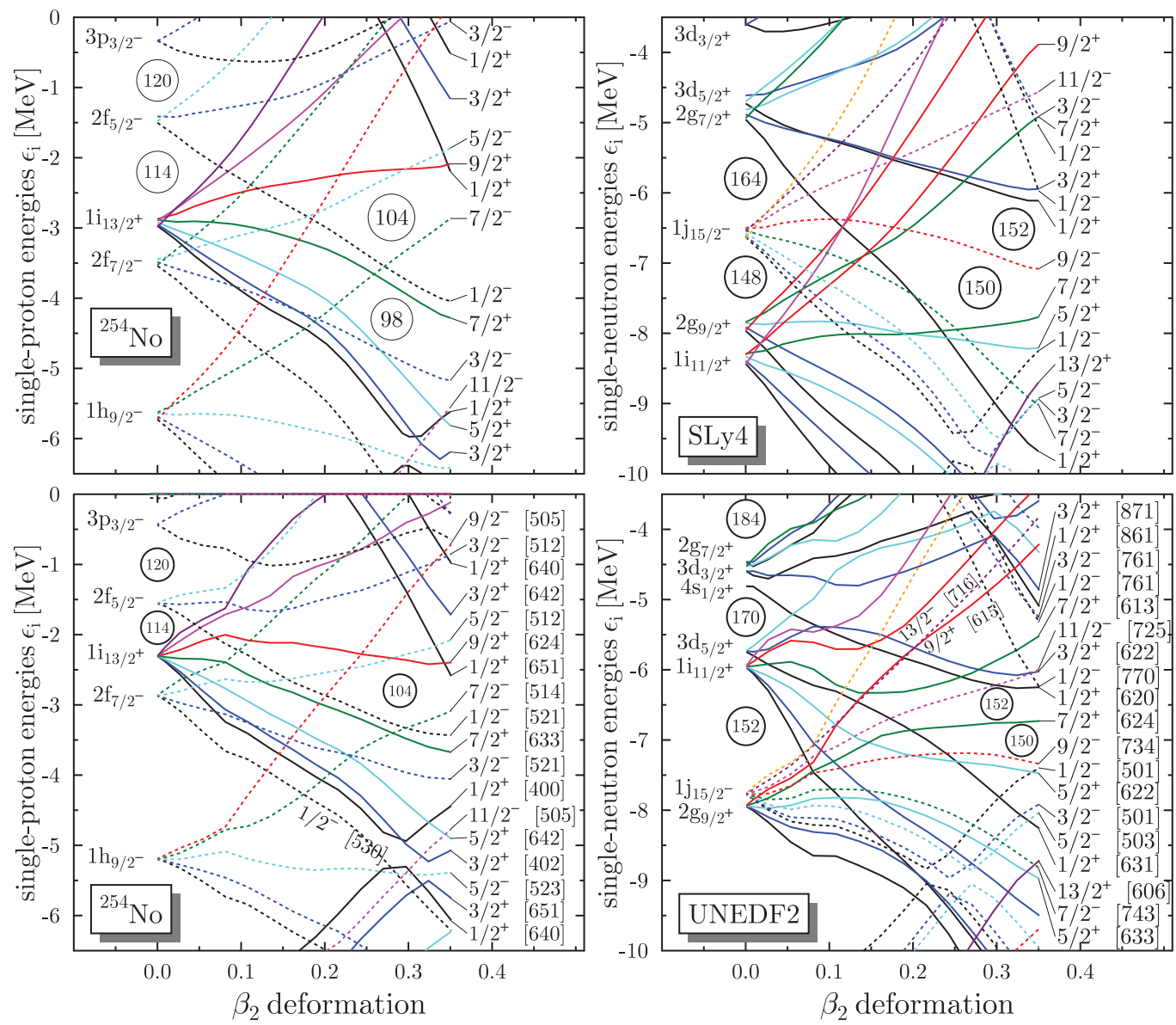

Figure 1: Proton (left panels) and neutron (right panels) Nilsson diagrams of ${ }^{254}$ No obtained for the Skyrme EDF SLy4 (upper panels) and UNEDF2 (lower panels). At spherical shapes, the orbitals are labelled with spherical quantum numbers. For SLy4, at large deformations, the deformed single-particle orbitals are labelled by the expectation values $\left\langle\hat{j}_{\|}\right\rangle$ of the projection of the angular momentum on the axial-symmetry axis. For UNEDF2, these orbitals are labelled by the Nilsson labels $\Omega\left[N n_{z} \Lambda\right]$ determined using code HFODD. Solid and dashed lines are used for the positive and negative parity states, respectively. 

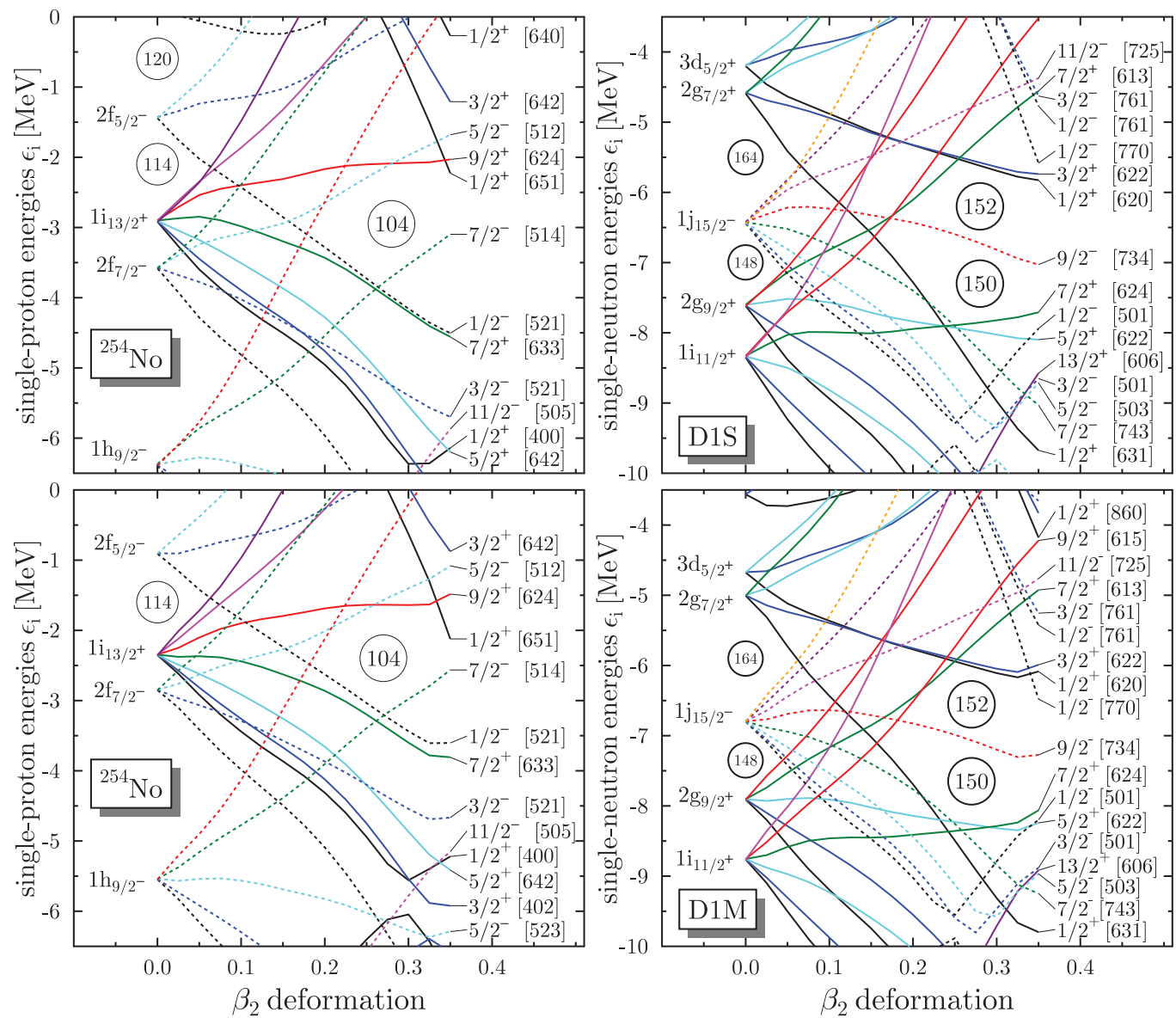

Figure 2: Same as in Fig. 1 but for the Gogny EDF D1S and D1M. All results and dominant Nilsson labels were determined using the axial-symmetry code HFBAXIAL. 

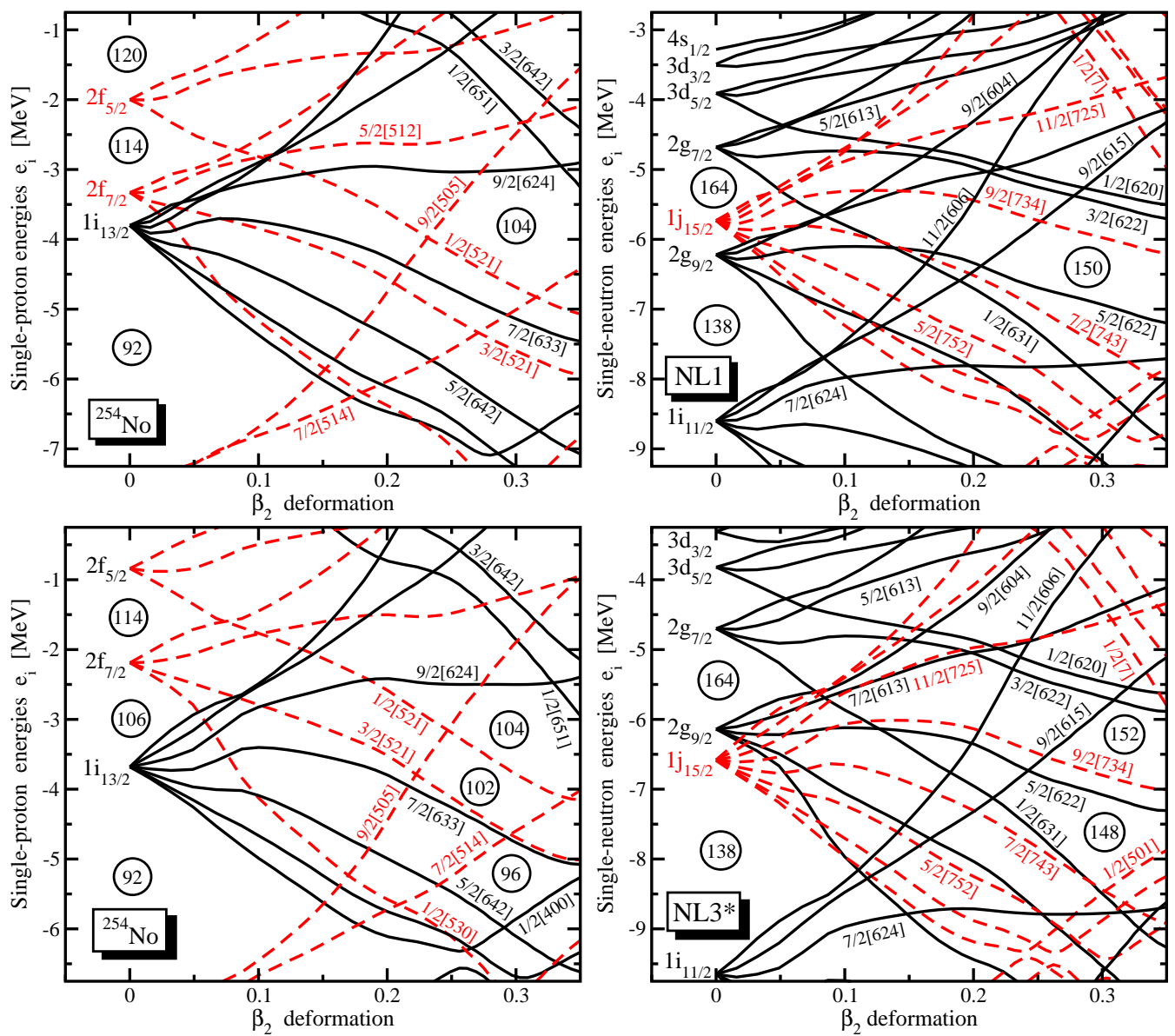

Figure 3: Same as in Fig. 1 but for the covariant EDFs NL1 and NL3*. All results and dominant Nilsson labels were determined using axially symmetric code RHB. 
in Fig. 1. Compared to SLy4, spherical orbital $1 j_{15 / 2^{-}}\left(1 i_{11 / 2^{+}}\right)$is lowered (raised) by about $2 \mathrm{MeV}$, and thus their relative positions are inverted, resulting in the spherical shell gaps at $N=152$ and 170, whereas other EDFs predict shell gaps at $N=164$.

The strong rearrangement of spherical neutron shells observed for UNEDF2 as compared to all other EDFs is a consequence of its rather large $C_{0}^{J J}$ and $C_{1}^{J J}$ coupling constants of the spin-current tensor terms of this parameterization [27]. In the $N \approx 152$ region, the inversion of the spherical level sequence substantially increases the number of filled spherical shells for which the spin-orbit partner is empty, thereby increasing the size of the spin-current terms. In fact, such behaviour is often found at mid-shell for parameterizations with large attractive tensor terms $[65,66]$.

The relativistic NL1 and NL3* functionals have the unique feature that they predict a large spherical $N=138$ gap of about $3 \mathrm{MeV}$ that is absent in all non-relativistic calculations. As the sequence of spherical subshells is different, for NL1 this gap is located between the $1 i_{11 / 2^{+}}$and $2 g_{9 / 2^{+}}$levels, whereas for NL3 ${ }^{*}$ it is found between the $1 i_{11 / 2^{+}}$and $1 j_{15 / 2^{-}}$levels.

\subsection{Quasiparticle spectra in ${ }^{251} \mathrm{Cf}$ and ${ }^{249} \mathrm{Bk}$}

In Fig. 4, calculated spectra of low-lying band-heads in ${ }^{249} \mathrm{Bk}$ and ${ }^{251} \mathrm{Cf}$ are shown along with the experimental data. In these results, the spectra were obtained by individually blocking relevant quasiparticle orbitals and then plotting differences of total many-body energies of obtained nucleonic configurations with respect of the total energy of the ground state, that is, they are not at all equivalent to quasiparticle energies understood as eigenvalues of the HFB Hamiltonian. The ground states were identified as nucleonic configurations with the lowest total energies.

Nuclear configurations of deformed odd nuclei (one-quasiparticle configurations) were labelled by means of the standard asymptotic quantum numbers $\Omega\left[N n_{z} \Lambda\right]$ (Nilsson quantum numbers) that correspond to the dominant component in the wave function of the blocked quasiparticle state.

We used the convention of plotting positive (negative) values for the excitation energies of quasiparticle configurations that correspond to blocked quasiparticle states having norms of the second HFB components smaller (larger) than $1 / 2$ before blocking. In this way, the states that are predominantly of a particle (hole) character appear above (below) zero energy. Moreover, these states are always plotted relatively to the ground-state; thus the 


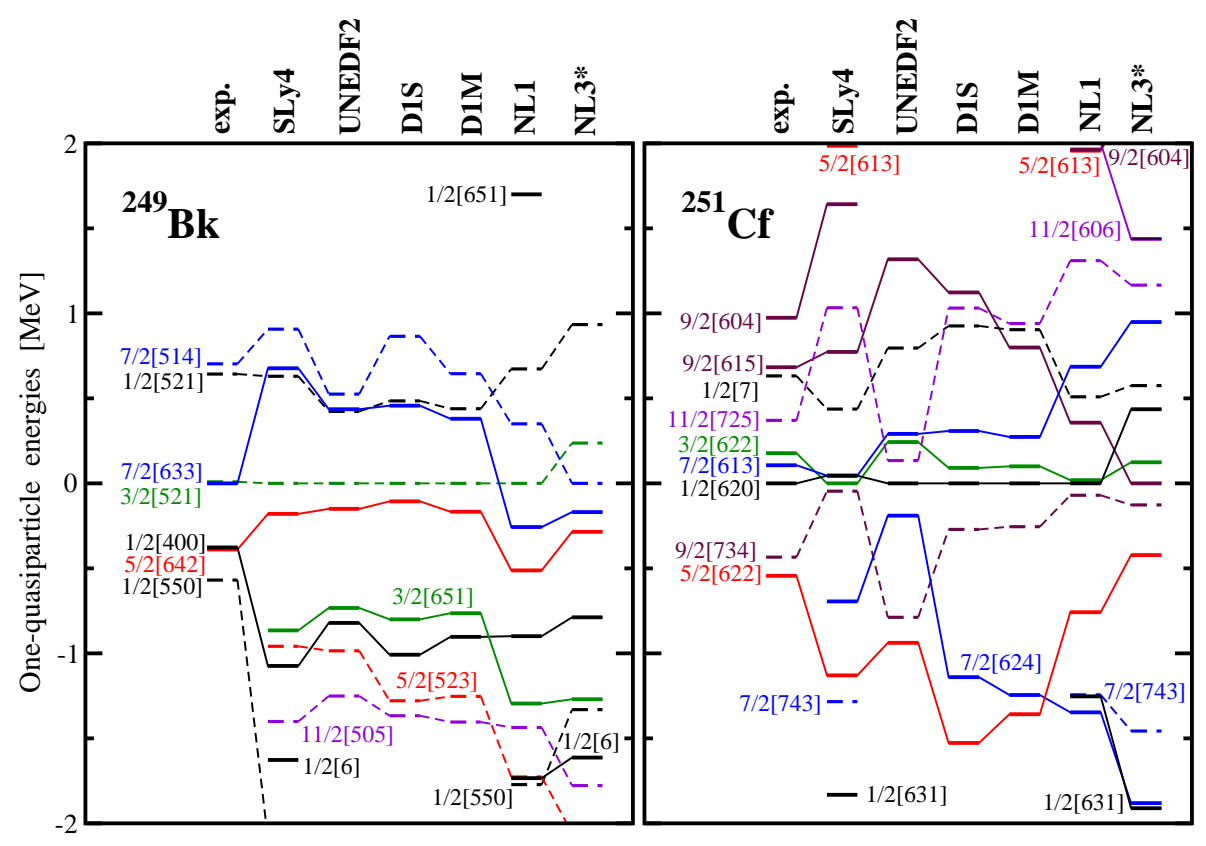

Figure 4: Experimental and calculated quasiparticle spectra in ${ }^{249} \mathrm{Bk}$ and ${ }^{251} \mathrm{Cf}$, see text for the convention used here. Experimental data are taken from Ref. [67] We label the state with the full Nilsson label of the dominant component of the wave function only if the squared amplitude of this component exceeds $50 \%$. The exception is the $1 / 2[7]$ state which is strongly mixed. However, the cumulative squared amplitude of the components of the wave function with $N=7$ in the structure of this state exceeds $90 \%$. Thus, we label it only by principal quantum number $N$ and $\Omega$. 
ground state is plotted identically at the value of zero energy. This convention facilitates the comparison of fully self-consistent results with the Nilsson diagrams. For experimental quasiparticle configurations, we follow the assignments of particle/hole character as presented in Figs. 34 and 35 of Ref. [68].

In these two odd nuclei, prominent intruder configurations correspond to the proton $7 / 2[633]$ and neutron $11 / 2[725]$ orbitals. In Ref. [21], these two orbitals were used as benchmark states to adjust strengths of the spinorbit interactions. We see that without such an adjustment, none of the studied standard EDFs places them at the right position. The ground-state proton $7 / 2[633]$ orbital, which experimentally is almost degenerate with the 3/2[521] orbital, for Skyrme and Gogny EDFs appears about $500 \mathrm{keV}$ above the ground state and for covariant EDFs about $200 \mathrm{keV}$ below the ground state, with the calculated ground states corresponding the $3 / 2[521]$ orbital (or $7 / 2[514]$ for the covariant EDF NL3*).

The neutron 11/2[725] orbital, for covariant, Gogny, and SLy4 EDFs, appears too high and for UNEDF2 EDF too low above its experimental position with respect to the ground-state $1 / 2[620]$ orbital. On the one hand, one can say that on the absolute scale these deficiencies are not large. On the other hand, they may point to slightly incorrect positions of spherical intruder orbitals, from which one would like to infer the shell structure of as yet not-reached superheavy nuclei. This analysis shows that detailed structure of very heavy deformed nuclei may depend on extremely fine details of the present-day theoretical models, which very well may be far beyond any reasonable possibility of adjusting them precisely enough to available experimental data.

Similarly as in the analysis presented in Ref. [21], as an attempt to improve the agreement with the experimental values, we have considered variations of the spin-orbit parameter $W_{L S}$ of the Gogny EDF D1S that could influence relative positions of intruder states. Increasing $W_{L S}$ from its nominal value of $130 \mathrm{MeV} \mathrm{fm}^{5}$ reduces the excitation energy of the $11 / 2^{-}$state while it increases the excitation energy of the $9 / 2^{-}$state in ${ }^{251} \mathrm{Cf}$. These changes improve the agreement with experimental data for larger values of $W_{L S}$. In the ${ }^{249} \mathrm{Bk}$ case, the $7 / 2^{+}$goes down in excitation energy as $W_{L S}$ increases, while the $5 / 2^{+}$and $1 / 2^{-}$levels go up. As in the ${ }^{251} \mathrm{Cf}$ case, the comparison with experiment seems to favor larger values of $W_{L S}$. This, however, has to be contrasted with the analysis of the shell structure of heavy spherical nuclei like ${ }^{208} \mathrm{~Pb}$, which usually calls for weaker spin-orbit interaction [25, 63, 65]. 


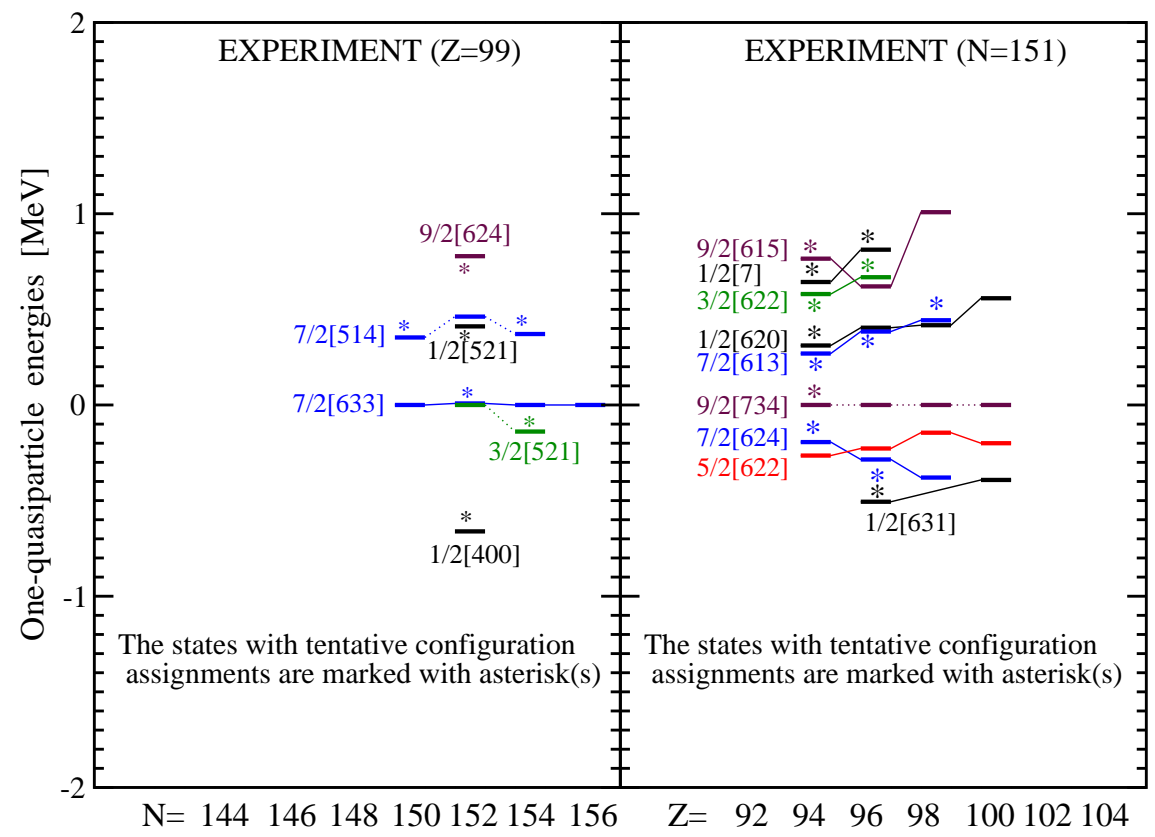

Figure 5: Experimental spectra [67] of the even- $N Z=99$ isotopes (left panel) and even- $Z$ $N=151$ isotones (right panel).

However, it is necessary to recognize that the studies restricted to spinorbit potential may have internal limitations that come from the fact that possible deficiencies in the description of the energies of the single-particle states emerging from the central potential, as for example those inferred in Ref. [40], are ignored. The fact that standard Skyrme functionals provide better description of the single-particle states in the $Z=115$ nuclei than the ones with the strength of spin-orbit interaction adjusted to experimental data in nobelium region [64] may be related to such limitation.

To explore the sensitivity of the results to the amount of pairing correlations in the system, for the Gogny EDFs we performed calculations where the pairing strengths of protons and neutrons were multiplied by factors $f_{p}$ and $f_{n}$, respectively. The first noticeable fact is that increasing the neutron pairing strength does not influence in a significant way the spectrum of ${ }^{249} \mathrm{Bk}$ (odd $Z$ ) as it also happens when increasing the proton pairing strength in ${ }^{251} \mathrm{Cf}$. Increasing $f_{p}$ reduces the excitation energy of all levels except for the $5 / 2^{+}$state that remains more or less constant. The comparison with experimental spectra seems to favor larger proton pairing correlations. In 
the ${ }^{251} \mathrm{Cf}$ case, all the levels except the lowest $3 / 2^{+}$decrease their excitation energy with increasing pairing strength. As in the ${ }^{249} \mathrm{Bk}$ case, increasing the pairing correlations in ${ }^{251} \mathrm{Cf}$ improves the agreement with experimental spectra. The same behavior was observed during the readjustment of the pairing strength for SLy4. However, on should keep in mind that pairing strengths are predominantly defined by the odd-even mass staggering, see Sect. 3.4 below.

\subsection{Quasiparticle spectra in $Z=99$ (Es) isotopes and $N=151$ isotones}

In the left (right) panels of Figs. 5-8, we show experimental and calculated spectra of the even- $N Z=99$ isotopes (even- $Z N=151$ isotones). The results are presented in the convention discussed in Sec. 3.2, with experimental assignments taken from Refs. [67, 68]. We see that the experimentally assigned odd-quasiparticle configurations are theoretically always obtained at relevant low excitation energies. However, similarly as in the case of ${ }^{249} \mathrm{Bk}$ and ${ }^{251} \mathrm{Cf}$ presented above, the details of the level spacing and ordering vary from one calculation to another and are not very well reproduced.

Although in this region of nuclei, the experimental information is richest in the particular isotopic and isotonic chains studied here, it is still quite scarce, and often experimental assignments of configurations are still only tentative [67]. Nevertheless, we can already see several conspicuous experimental trends.

In protons, we see the $7 / 2[633]$ ground states and $7 / 2[514]$ excited states at fairly constant excitation energies of about $400 \mathrm{keV}$. For the Skyrme EDF SLy4, this feature is very well reproduced, with a significant drop of this excitation energy predicted at $N=156$. For the Skyrme EDF UNEDF2, the $7 / 2[514]$ level is also obtained above the Fermi level, with the excitation energy gradually decreasing already at $N=152$. Note, however, that for the Skyrme EDFs, in ${ }^{249} \mathrm{Bk}$ the relative positions of these two levels were not very precisely reproduced, so the nice agreement obtained in the $Z=99$ isotopes might be fortuitous. For the Gogny EDFs, this pair of the levels is obtained at roughly correct excitation energies, whereas for the covariant EDFs, these levels are fairly well degenerate.

In neutrons, the 9/2[734] ground states are for all EDFs studied here well reproduced, apart from the Skyrme EDF UNEDF2, which gives the $7 / 2[613]$ ground state with the hole-character 9/2[734] orbitals appearing at excitation energy of about $400-500 \mathrm{keV}$. Two particle-character excited quasiparticle states, $7 / 2[613]$ and $1 / 2[620]$, show excitation energies which 

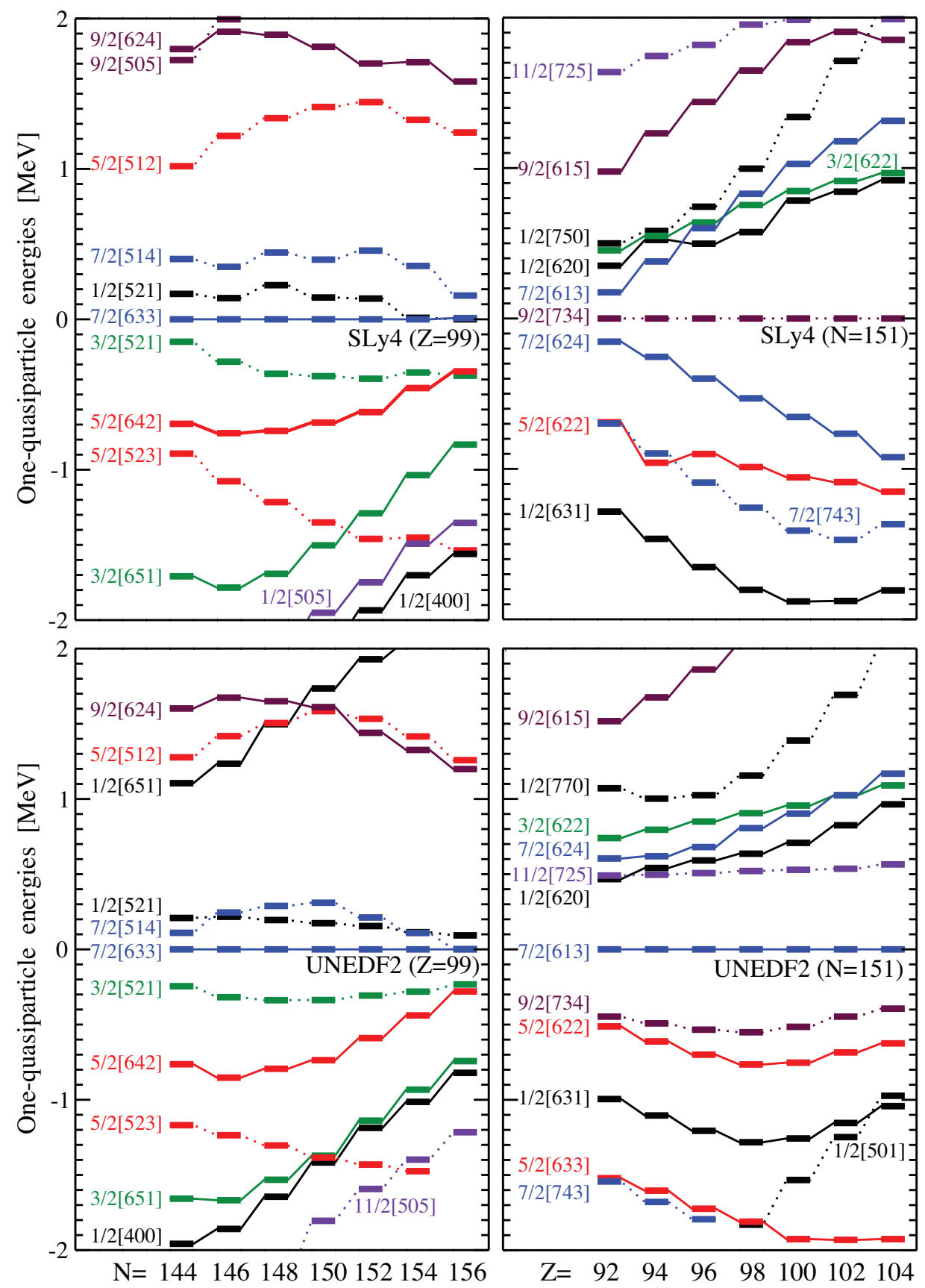

Figure 6: Same as in Fig. 5 but for the spectra calculated for the Skyrme EDF SLy4 (upper panels) and UNEDF2 (lower panels). Since for SLy4 only the mean values of the single-particle angular momenta $\left\langle\hat{j}_{\|}\right\rangle$and $\left\langle\hat{j}^{2}\right\rangle$ (and not full Nilsson labels) were available, in this case the labels were assigned by analogy with the results obtained for the Gogny EDF D1S, which provides very similar spectra. 

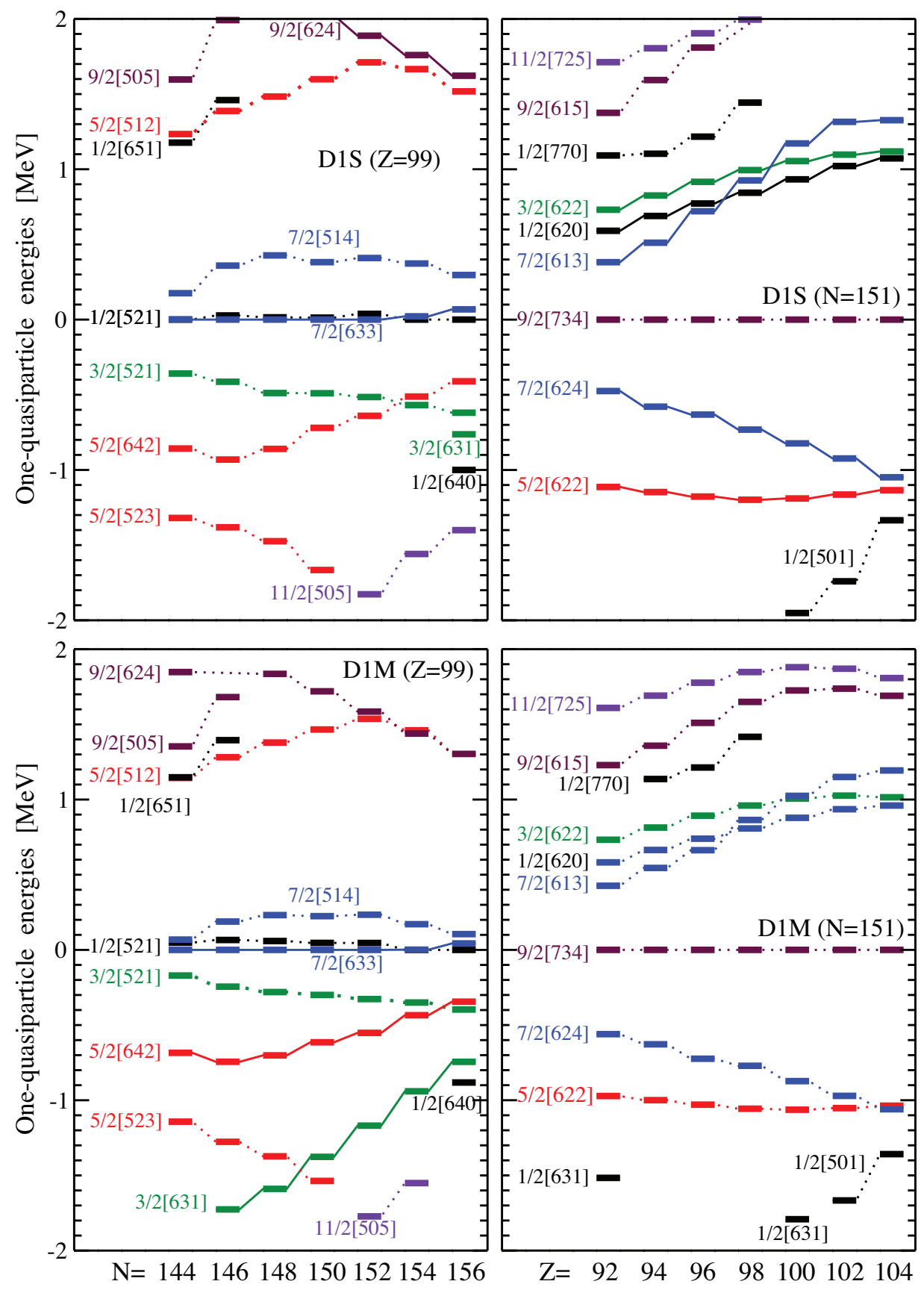

Figure 7: Same as in Fig. 5 but for the spectra calculated for the Gogny EDF D1S (upper panels) and D1M (lower panels). 

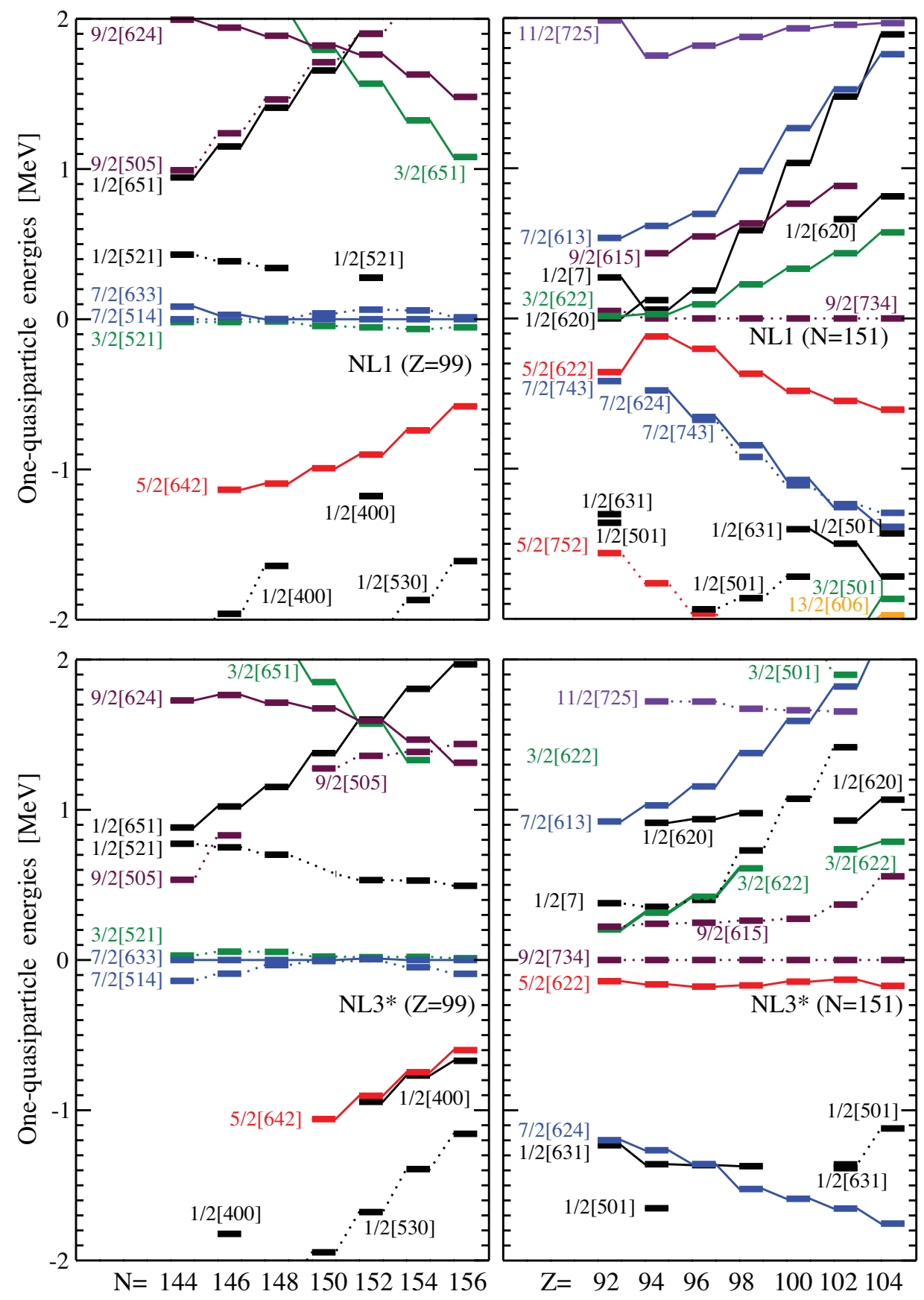

Figure 8: Same as in Fig. 5 but for the spectra calculated for the covariant EDF NL1 (upper panels) and NL3* (lower panels). 
increase with mass. They are correctly reproduced for the Skyrme SLy4 and covariant EDF's; however, the increase with mass is too fast. Correct trends of these two levels are also determined for the Gogny EDFs. Two other hole-character experimental quasiparticle states, 7/2[624] and 5/2[622], have excitation energies weakly increasing and decreasing with mass, respectively. For the Skyrme SLy4 and covariant NL1 EDFs, their excitations energies both increase quite rapidly with mass, whereas for the Gogny functionals the mass dependence is rather correct. In all models studied here, the energy splitting of these two levels is too large as compared to data.

From the systematics of the one-quasiparticle levels one can draw a number of interesting conclusions about the significance of the Nilsson diagram of single-particle levels for the calculated and observed spectra of one-quasiparticle levels. Already in schematic models there is no quantitative one-to-one correspondence, as the presence of pairing correlations modifies the spectrum of low-lying states. In nuclear EDF calculations, there are additional selfconsistency effects from the separate optimization of each one-quasiparticle state.

As already outlined in Sect. 3.1, the various EDFs do not always agree on the size of the energy gaps between spherical subshells in the Nilsson diagrams for ${ }^{254}$ No plotted in Figs. 1, 2 and 3, sometimes even not on the sequence of the levels. For example, the relativistic NL1 and NL3* functionals predict a large spherical $N=138$ gap of about $3 \mathrm{MeV}$. For the non-relativistic D1S, D1M, and SLy4 EDFs this gap is much smaller and even disappears for UNEDF2. With the non-relativistic functionals one finds spherical gaps at $N=148$ and $N=152$ instead. The appearance and size of the $N=$ 138 gap is controlled by the position of the spherical $1 i_{11 / 2^{+}}$level below and the $2 g_{9 / 2^{+}}$level above, except for NL3* for which the $1 j_{15 / 2^{-}}$is pulled below the $2 g_{9 / 2^{+}}$. The neutron $9 / 2[734], 5 / 2[622]$, and $9 / 2[615]$ single-particle levels that emerge from the spherical $1 j_{15 / 2-}, 2 g_{9 / 2^{+}}$, and $i_{11 / 2^{+}}$subshells, respectively, are found just above and below the deformed $N=150$ and $N=152$ gaps in the Nilsson diagrams for ${ }^{254}$ No. Looking at one-quasiparticle spectra of ${ }^{251} \mathrm{Cf}$, as plotted in Fig. 4, one finds that for SLy4, D1S, and D1M the distance between the hole-character 9/2[734] and particle-character $9 / 2[615]$ levels is very satisfactorily described within $200 \mathrm{keV}$, whereas for UNEDF2 it is much too large, and for NL1 and NL3* it is much too small. On the other hand, in the $N=151$ isotones shown in Figs. 5-8, the distance between these two levels is on average too large for SLy4, D1S, and D1M, again much too large for UNEDF2, correct for NL1, and again much too 
small for NL3*. This indicates that the spherical $N=138$ gap of NL3 ${ }^{*}$ is probably too large. Still, it is unlikely that it has to be made as small as the one found for Skyrme and Gogny EDFs.

By contrast, the distance between the 9/2[734] and 5/2[622] one-quasiparticle levels in ${ }^{251} \mathrm{Cf}$ and $N=151$ isotones, which are connected to the $1 j_{15 / 2^{-}}$and $2 g_{9 / 2^{+}}$shells, respectively, is overestimated by almost $1 \mathrm{MeV}$ for SLy4, D1S, and D1M, by a few hundred keV for NL1 and UNEDF2, but quite well reproduced by NL3*. This indicates first of all that the pronounced deformed $N=150$ gap visible in the Nilsson diagrams for SLy4, D1S, D1M, and NL1 should be much smaller. In fact, the large deformed gap at $N=150$ that is predicted by a substantial number of nuclear EDFs has already quite often been attributed to be one of the main causes for the disagreement between calculated and observed spectroscopic properties of nuclei in the $A \approx 250$ mass region $[1,8,21,40,43]$. It is remarkable that the UNEDF 2 and NL3* functionals that both describe well the distance between the 9/2[734] and $5 / 2[622]$ levels give very different shell structure at spherical shape in the Nilsson diagrams of Figs. 1 and 3. For UNEDF2, the spherical $1 j_{15 / 2}$ - shell is about $500 \mathrm{keV}$ above the spherical $2 g_{9 / 2^{+}}$, whereas for NL3* it is the other way round, the latter being a unique feature among the functionals studied here. Note that for NL3* the relative position of these levels is quickly moving with particle number: for the slightly lighter ${ }^{244} \mathrm{Cm}$ the $1 j_{15 / 2-}$ is already above $2 g_{9 / 2}+$ level like for the other functionals studied here, cf. Ref. [20].

Altogether, these findings indicate that on cannot expect to find a unique one-to-one correspondence between the spectra of one-quasiparticle levels of deformed nuclei and spherical single-particle levels in a Nilsson diagram. Different shell structure in the Nilsson diagrams might lead to similar onequasiparticle spectra and vice versa. Indeed, the rearrangement, polarization, pairing and single-particle-mixing effects when constructing self-consistent one-quasiparticles state make the connection quite complex and apparently also slightly EDF-dependent. In addition, it should be recalled that for nuclei in the $A \approx 250$ region the spherical configuration corresponds to a maximum of the deformation energy landscape and therefore should not be associated with a physical state.

Similar cases can be found for proton levels. All Nilsson diagrams for ${ }^{254}$ No plotted in Figs. 1, 2 and 3 exhibit a substantial spherical $Z=92$ gap that is located between the $2 f_{7 / 2^{-}}$and $1 h_{9 / 2^{-}}$orbitals in non-relativistic functionals and between the $1 i_{13 / 2}$ and $1 h_{9 / 2}$ orbitals in covariant functionals. It has been pointed out in Refs. [69, 70] that there is no indication for such 
a gap, which is also visible in the single-particle spectra of lighter spherical nuclei [63], in the available spectroscopic data for the spherical ${ }^{216} \mathrm{Th}$ and ${ }^{218} \mathrm{U}$ nuclei. However, the $7 / 2[514], 3 / 2[521], 1 / 2[521]$, and $7 / 2[633]$ onequasiparticle levels of ${ }^{249} \mathrm{Bk}$ and the Es isotopes, which originate from the spherical $1 h_{9 / 2}, 2 f_{7 / 2}$ and $1 i_{13 / 2}$ subshells surrounding the spherical $Z=92$ gap, are reasonably well described by all EDFs employed here, see Figs. 4-8. As a consequence, a large spherical $Z=92$ gap in the Nilsson diagram of ${ }^{254} \mathrm{No}$ is not in apparent conflict with the available data for deformed nuclei in the $A \approx 250$ mass region. In particular the non-relativistic Skyrme and Gogny functionals describe well the relative position of the 3/2[512] and $7 / 2[514]$ levels within a few hundred keV. It is only for the relativistic NL1 and NL3* functionals that the spacing between the $7 / 2[514]$ and $7 / 2[633]$ one-quasiparticle levels becomes slightly too small, cf. Figs. 4 and 8, which points to a slight overestimation of the spherical $Z=92$ gap for these functionals. Still, the necessary shift of the spherical shells that one would expect to correct for the disagreement between calculation and data will not even reduce the gap to the size found with non-relativistic EDFs.

It is necessary to recognize that the present investigation represents one of first steps in the direction of understanding of the accuracy of the description of one-quasiparticle states in deformed nuclei, which, however, goes beyond previous attempts by directly comparing different classes of the EDF approaches for the same set of experimental data. Based on the current set of experimental data similar accuracy is achieved for employed EDF's. So far statistical analysis of the accuracy of the description of one-quasiparticle states employing full set of available experimental data on proton and neutron states has been performed only in actinides and only in the framework of CDFT using NL1 and NL3* EDF's in Ref. [13]. Although many of the states are described with acceptable accuracy, for some states the deviation of calculated energy from experimental one exceeds $1 \mathrm{MeV}$.

Less systematic studies have been performed in the non-relativistic EDF approaches. Global survey of the ground state configurations in odd-mass nuclei employing three Skyrme functionals has been performed in Ref. [71]. In Skyrme EDF, the spectra of few actinides and of odd-proton Ho nuclei have been studied in Refs. [7, 37]. The spectra of selected Rb, Y, and Nb nuclei have been studied in axial Gogny EDF in Refs. [72, 73].

These investigations reveal the same two sources of uncertainties [13]. Although the same set of single-particle states appear in the vicinity of the Fermi level as in experiment, their relative positions and energies are not 
always correct. This is first source of uncertainties. The second source of uncertainty is related to stretched energy scale in model calculations as compared with experiment which is due to low effective mass of the nucleon at the Fermi level.

While the solution of the first problem can be attempted in the EDF framework, the current analysis with different classes of EDF approaches suggest that it is not likely to remove all existing problems in the description of the single-particle spectra (see also the discussion in Ref. [13]). More comprehensive solution, which would also address the second source of uncertainty, would require taking into account particle-vibration coupling which will explain existing fragmentation of the single-particle states and possibly compress the calculated one-quasiparticle spectra bringing them closer to experiment. Combined with respective re-parametrization of the functionals it may lead to the functionals with better spectroscopic quality. It is clear that existing functionals are biased towards bulk properties since either no (CDFT) or extremely limited information on single-particle properties (Skyrme and Gogny EDF) is used in their fitting protocols. Even in this situation the calculated spectra are not far away from experiment which can be considered as a success and a good starting point for future development.

\subsection{Odd-even and two-particle mass staggering}

In order to analyze the odd-even mass differences, the three-point pairing indicators [74] (staggering parameters),

$$
\Delta_{p}^{(3)}=\frac{1}{2}(B(Z+1, N)+B(Z-1, N)-2 B(Z, N))
$$

with odd $Z$ and even $N$, and

$$
\Delta_{n}^{(3)}=\frac{1}{2}(B(Z, N+1)+B(Z, N-1)-2 B(Z, N))
$$

with even $Z$ and odd $N$, where $B(Z, N)$ is the (positive) binding energy of the nucleus, have been plotted in Figs. 9 and 10, respectively. In our HFB and RHB calculations, increased sizes of the shell gaps can, in principle, be seen in the odd-even mass staggering parameters (6) and (7), because the density of single-particle states has an immediate bearing on the calculated intensity of pairing correlations. This is under assumption that the differences obtained by blocking different orbitals along the isotopic or isotonic chains have a lesser impact on the odd-even mass staggering. Irrespective 


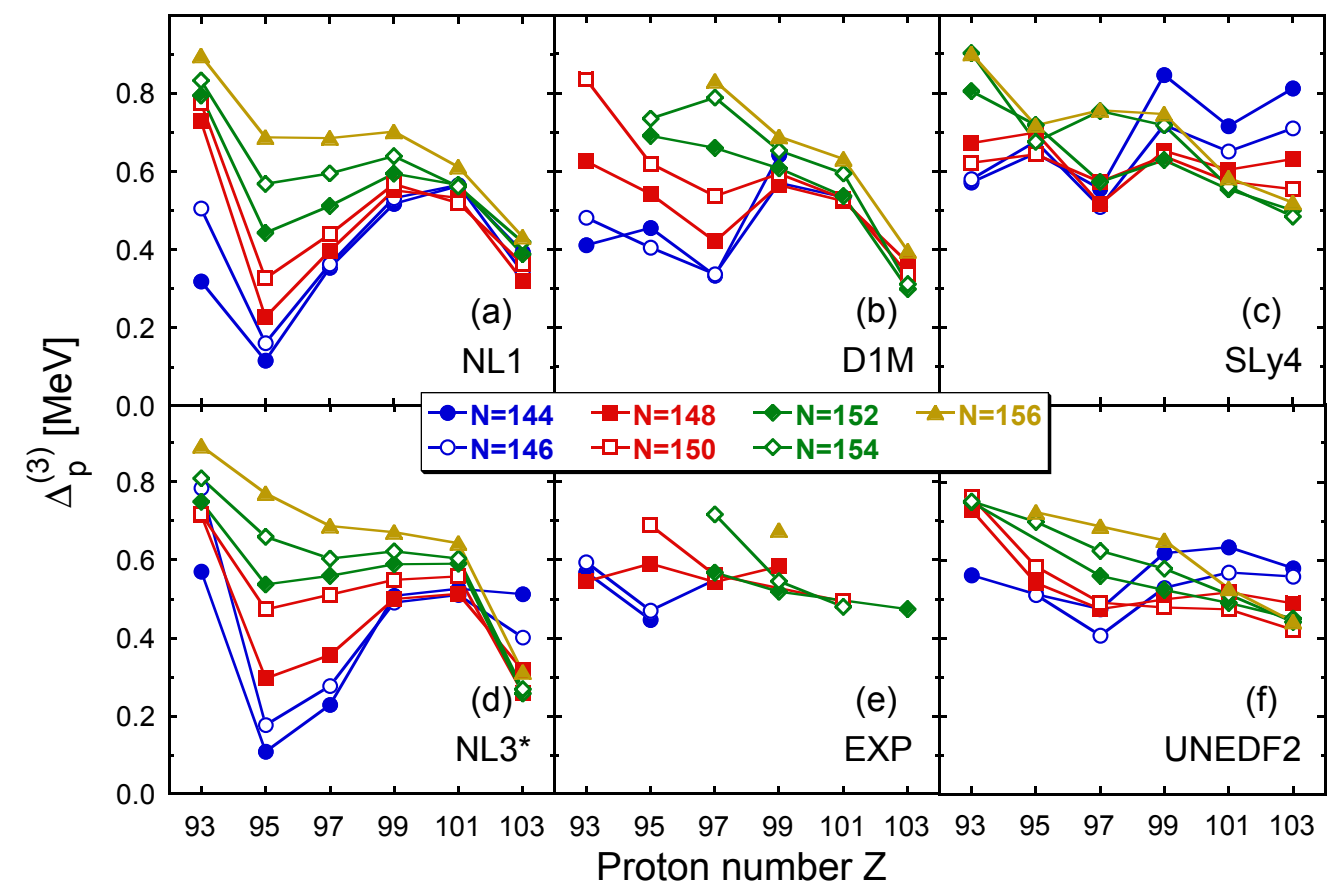

Figure 9: Three-point proton odd-even mass staggering, Eq. (6), shown for the odd- $Z$ and even- $N$ nuclei in the nobelium region. Experimental values are based on the AME2012 atomic mass evaluation [75].

of the detailed reproduction of the experimental values of these parameters, calculated results may thus illustrate the shell structure corresponding to the EDFs studied here.

Experimental results, shown in panels (e) of Figs. 9 and 10, indicate that in the nobelium region, values of the staggering parameters are within the range of 500-700 keV. On closer inspection, we see several trends in the mass dependence of these parameters, which may indicate variations of the shell structure due to the influence of the level density on pairing correlations, or due to other fine structural effects. In particular, values of $\Delta_{p}^{(3)}$ seem to have a small dip for the $N=146$ isotones at $Z=95$, are fairly constant in the $N=148$ isotones, and gradually decrease with mass in the $N=150-154$ isotones. None of these values indicate a particularly significant shell-gap opening near $Z=100$. Similarly, small dips in $\Delta_{n}^{(3)}$, which show up in the $Z=96-98$ isotopes at $N=149$ and in the $Z=100$ isotopes at $N=153$, do not point to a particularly large shell gap at $N=152$.

This lack of large variations in odd-even mass staggering is at variance 


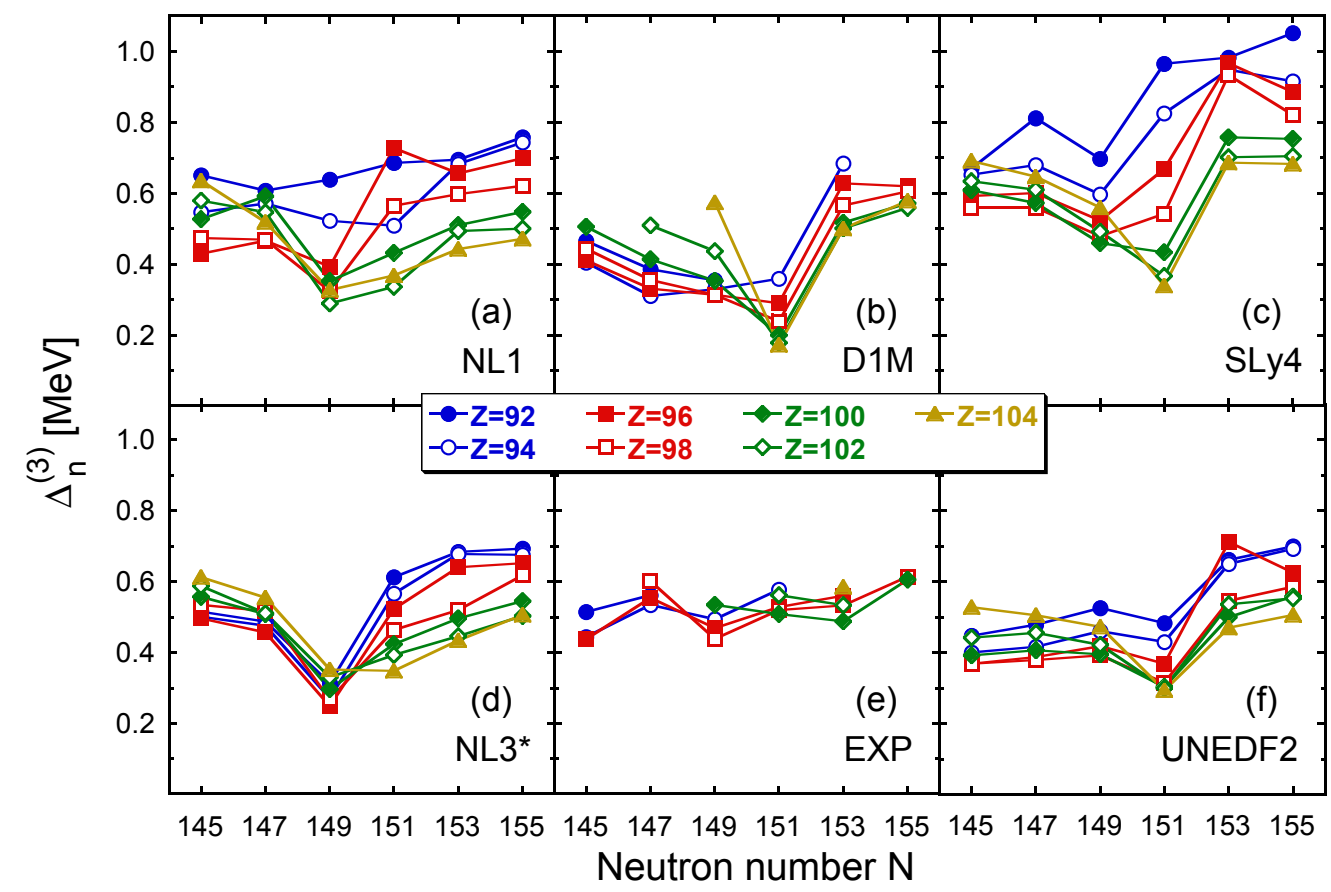

Figure 10: Same as in Fig. 9 but for the neutron odd-even mass staggering, Eq. (7), shown for the odd- $N$ and even- $Z$ nuclei.

with the analysis of two-particle mass staggering given by quantities

$$
\delta_{2 p}^{(3)}=2 B(Z, N)-B(Z+2, N)-B(Z-2, N)=S_{2 p}(Z, N)-S_{2 p}(Z+2, N)
$$

and

$$
\delta_{2 n}^{(3)}=2 B(Z, N)-B(Z, N+2)-B(Z, N-2)=S_{2 n}(Z, N)-S_{2 n}(Z, N+2),
$$

which were typically used to identify two-nucleon shell gaps in experiment and in calculations for spherical $[62,76]$ and deformed $[77,8]$ shell closures in the predictions of Skyrme and covariant EDFs. As discussed in Ref. [2], experimental values of $\delta_{2 n}^{(3)}$ show clear maxima for the $Z=96-102$ isotopes at $N=152$ and those of $\delta_{2 p}^{(3)}$ exhibit maxima for the $N=148-150$ isotones at $Z=98$ and for the $N=152-154$ isotones at $Z=100$, see also Figs. 11 and 12 .

When looking at the most pronounced features of the calculated oddeven mass staggering shown in Figs. 9 and 10, we see that minima of $\Delta_{p}^{(3)}$ can be seen at $Z=95$ (NL1 and NL3* EDFs) and $Z=97$ (D1M, SLy4, 


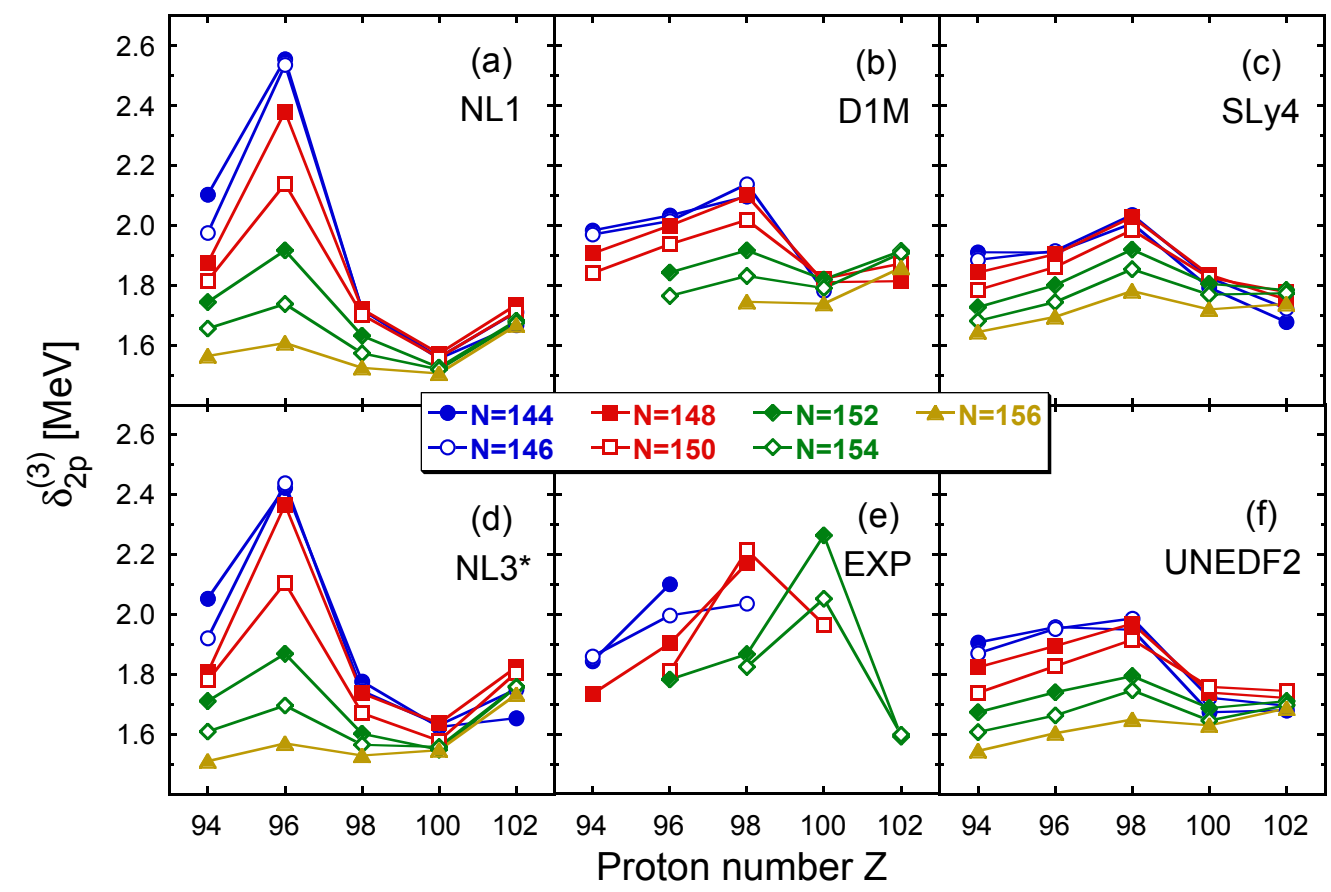

Figure 11: Two-proton mass staggering, Eq. (8), shown for the even- $Z$ and even- $N$ nuclei in the nobelium region. Experimental values are based on the AME2012 atomic mass evaluation [75].

and UNEDF2 EDFs). For the Gogny and Skyrme EDFs, these minima disappear at higher neutron numbers and rather monotonic trends are then obtained. Similarly, minima of $\Delta_{n}^{(3)}$ appear at $N=149$ (NL1 and NL3* EDFs) or $N=151$ (D1M, UNEDF2, and SLy4 EDFs); in the latter case, in lighter isotopes they tend to shift to $N=149$. For the calculated twoproton-staggering indicators (8), covariant EDFs, NL1 and NL3*, exhibit very strong maxima at $Z=96$, at variance with the data, whereas the nonrelativitic EDFs, D1M, SLy4, and UNEDF2, reproduce experimental maxima at $Z=98$ in the $N=146-150$ isotones but fail to shift these maxima to $Z=100$ in heavier isotones. This conspicuous experimental fearure thus remains unsolved. The calculated two-neutron-staggering indicators (9), do not reproduce experimental maxima occurring at $N=152$. These results illustrate the fact that none of the studied EDFs reproduces the experimental trends in shell gaps extracted from the two-particle indicators (8) and (9). We note here that the inclusion of the LN method into the calculations renders pairing correlations much less sensitive to the shell structure. Therefore, one 


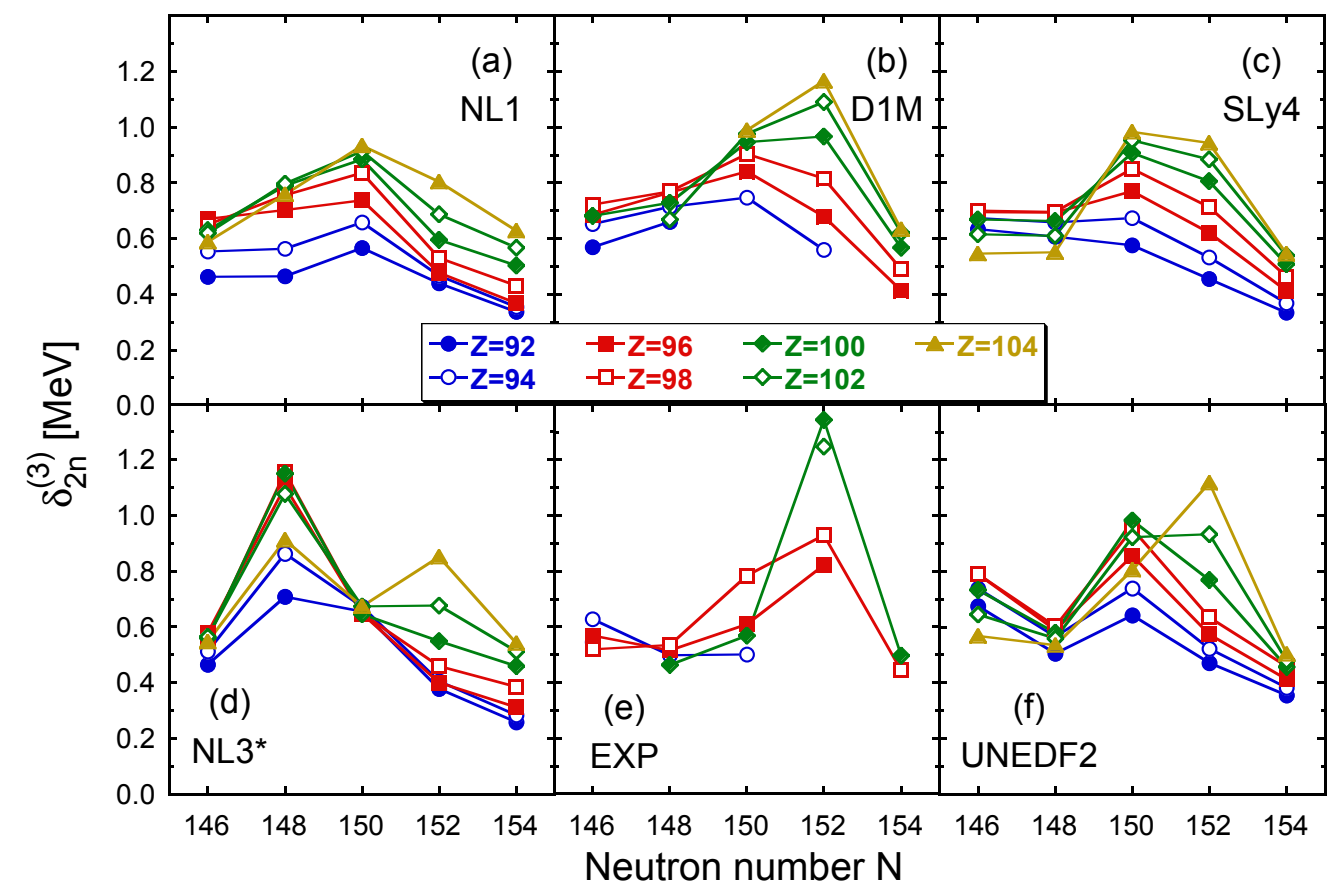

Figure 12: Same as in Fig. 11 but for the two-neutron mass staggering, Eq. (9).

then obtains fairly structureless trends of $\Delta_{p}^{(3)}$ and $\Delta_{n}^{(3)}$ [21], although for covariant EDFs, one at the same time obtains a significant improvement of the overall agreement with experimental values [20].

\subsection{Moments of inertia}

The moments of inertia may constitute another independent indicator of the shell structure. This is true under the assumption that their values, similarly as for the odd-even staggering, are dictated by varying pairing correlations. Then, larger shell gaps would induce weaker pairing and thus larger moments of inertia.

The kinematical moments of inertia were calculated as

$$
J^{(1)} \equiv \frac{\left\langle\hat{J}_{\perp}\right\rangle}{\omega_{\perp}},
$$

where $\omega_{\perp}$ is the value of the constrained rotational frequency and $\left\langle\hat{J}_{\perp}\right\rangle$ is the expectation value of the component of angular momentum, both in the directions perpendicular to the axial-symmetry axis. The dynamic moment 
of inertia is not considered due to larger experimental uncertainties consequence of higher order derivatives in its definition. Moreover, contrary to the kinematic moment of inertia the dynamic one does not depend on spin. Thus, the kinematic moment of inertia provides stricter constrain of model predictions.

All calculations of moments of inertia were performed at $\hbar \omega_{\perp}=20 \mathrm{keV}$. In Figs. 13 and 14, they are compared to experimental values determined as

$$
J^{(1)} \equiv \frac{3 \hbar^{2}}{E_{2^{+}}} .
$$

In nuclei where at the bottom of rotational bands the experimental $2^{+}$levels have not been seen, we used values extrapolated [78] by the Harris formula. In this region of nuclei, the low-spin moments of inertia turn out to be very weakly dependent on the angular frequency, and, therefore, a specific method of extracting them from experiment is not essential.

The experimental values shown in panels (e) of Figs. 13 and 14 show clear maxima of $J^{(1)}$ in function of $Z$ at $Z=100$ in the $N=152$ isotonic chain, and at $Z=98$ in the $N=150$ isotonic chain, as well as function of $N$ at $N=152$ in the $Z=100$ isotopic chain. These maxima only partly appear in those nuclei that show maxima of the two-particle staggering indicators, discussed in Sec. 3.4.

In our theoretical calculations, weak maxima of $J^{(1)}$ are obtained at $Z=$ 98 for $N=146-150$ isotonic chains (D1M ${ }^{1}$, UNEDF2, and SLy4 EDFs) and a stronger maxima at $Z=96$ for $N=146-152$ isotonic chains (NL1 and NL3* EDFs), whereas in heavier isotonic chains we see only a gradual increase, without indications of increased shell gaps. Similarly, in the isotopic chains maxima appear at $N=148$ (NL1 EDF) and merely kinks appear at $N=150$ (D1M, UNEDF2, and SLy4 EDFs)

Comparing moments of inertia, Figs. 13 and 14, with the Nilsson diagrams, Figs. 1-3, we see that our calculations with different models and forces seem to exhibit rather nice correspondence between the respective proton (neutron) single-particle shell gaps and peaks/kinks in the moments of inertia along the isotonic (isotopic) chains.

In Fig. 13, for the covariant EDFs NL1 (a) and NL3* (d), the peaks at $Z=96$ obtained for the $N=144-152$ isotonic chains can be associated with

\footnotetext{
${ }^{1}$ Results for D1S can be found in Ref. [10]
} 


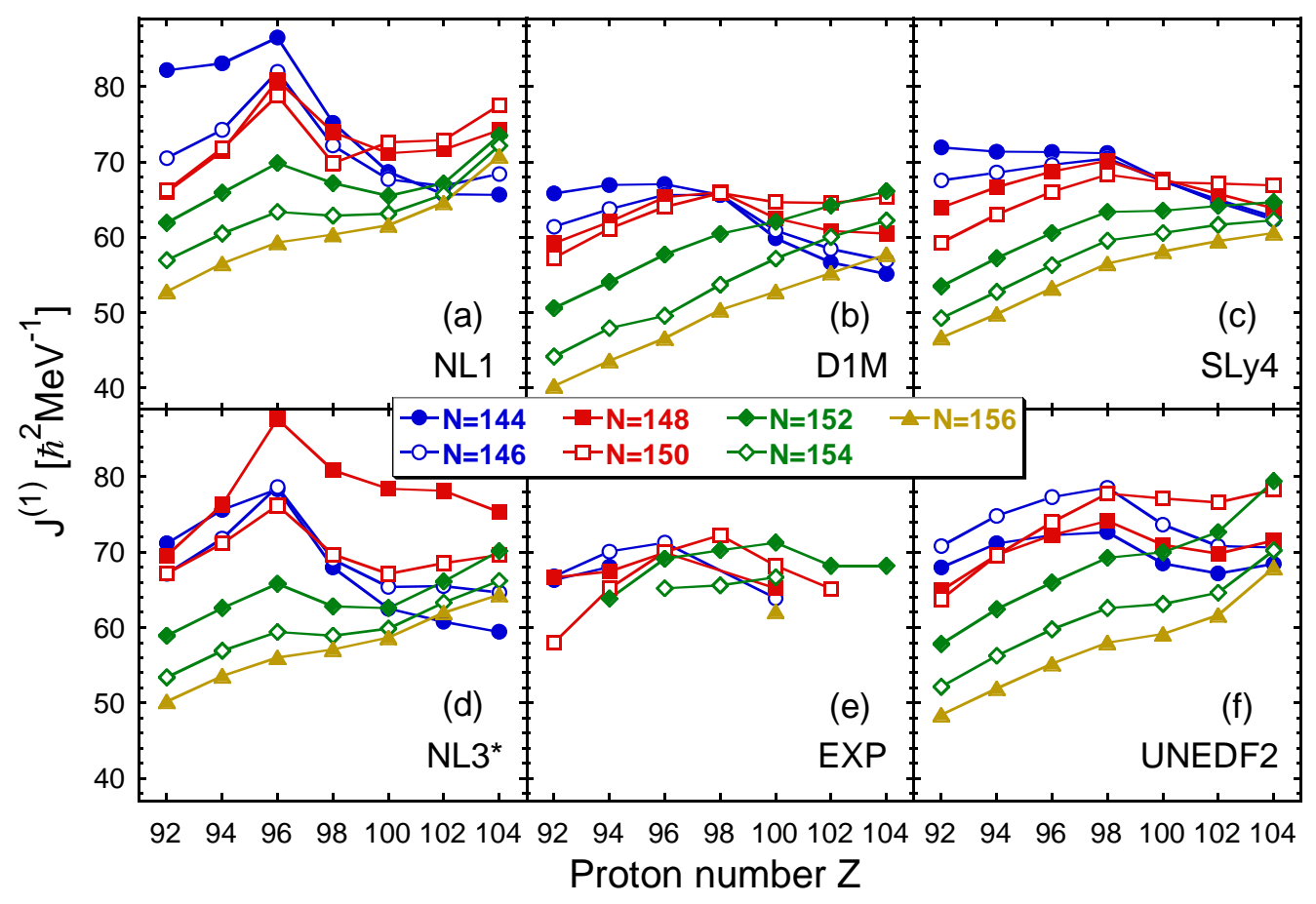

Figure 13: Kinematical moments of inertia $\mathcal{J}^{(1)}$ of yrast rotational bands of even-even nuclei in the nobelium region plotted in the isotonic chains as functions of $Z$. Theoretical values, calculated at the angular frequency of $\hbar \omega_{\perp}=20 \mathrm{keV}$, Eq. (10), are compared to experimental values extracted from energies of the $2^{+}$states, Eq. (11). Experimental energies are taken from Ref. [67] except for ${ }^{252} \mathrm{Fm}$, which is taken from Ref. [79].

the shell gap that in Fig. 3 opens up at $Z=96$. The peak moves to $Z=104$ for $N=154$ and 156 chains, see Fig. 3. For the Skyrme EDFs SLy4 (c) and UNEDF2 (f), the peak/kink at $Z=98$ may be associated with a shell gap at $Z=98$ visible in the Nilsson diagram of Fig. 1. For the Gogny EDF $\mathrm{D} 1 \mathrm{M}$, the correspondence is not as clear as that visible in other cases. It is to be noted that all our calculations predict that for most of the isotonic chains, values of $\mathcal{J}^{(1)}$ peak at $Z=104$. This corresponds to the proton gap at $Z=104$ that is clearly visible in Figs. $1-3$.

In Fig. 14, for the covariant EDF NL3* (d), values of $\mathcal{J}^{(1)}$ show pronounced maxima at $N=148$ for isotopic chains of $Z=96-104$. For NL1 (a), the maximum becomes a kink occurring at $N=150$. Neutron numbers of $N=148$ and 150 correspond nicely to the shell gaps shown in Fig. 3. Non-relativistic functionals predict either a peak at $N=150$ or a plateau for 


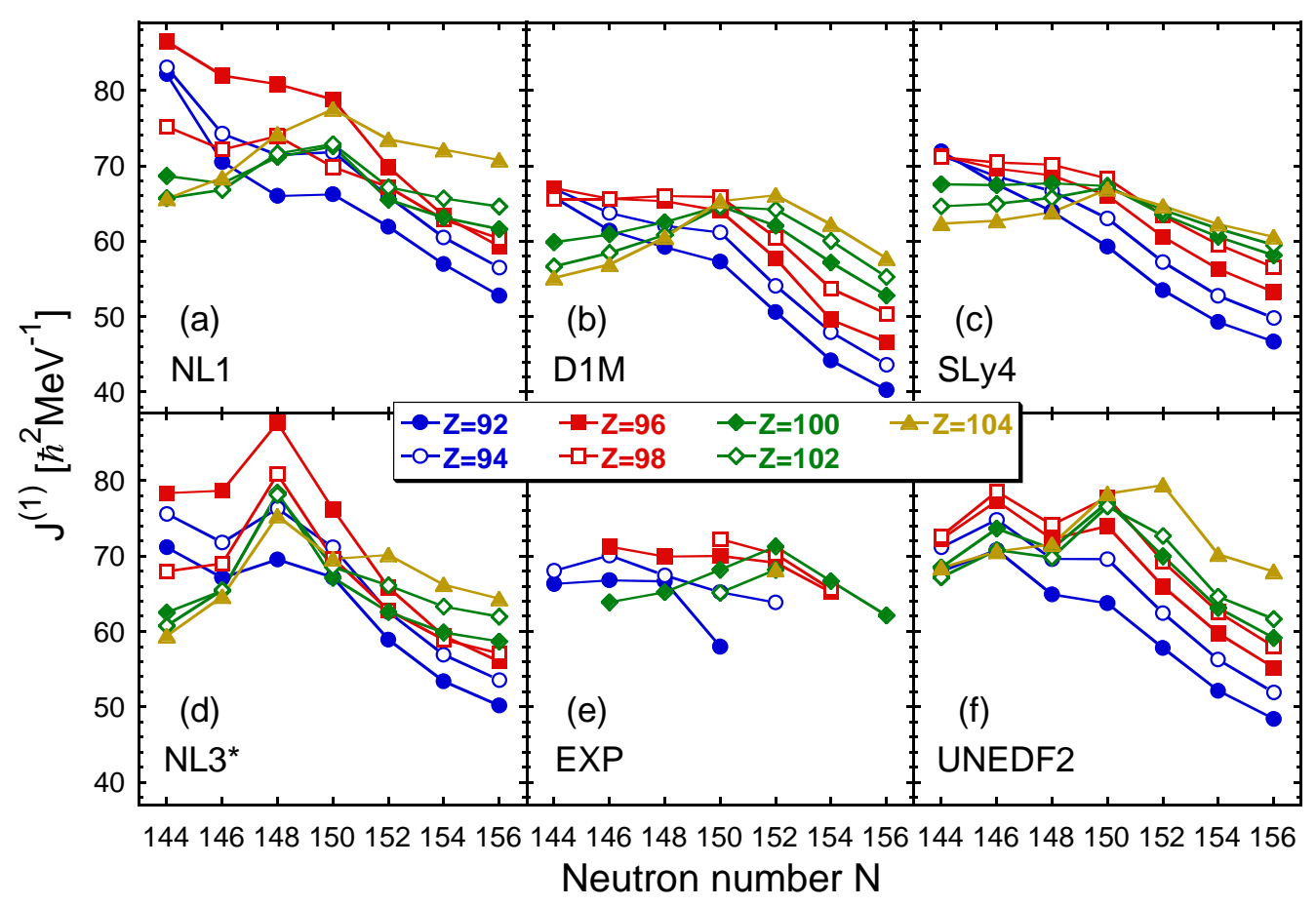

Figure 14: Same as in Fig. 13, but plotted along the isotopic chains as functions of $N$.

$N=150$ and 152 , particularly for the $Z=100-104$ isotopic chains, shown in Fig. 14.

It is necessary to recognize that for a proper reproduction of experimental moments of inertia, the inclusion of the LN method into the calculations appears to be more important in covariant [80] than in non-relativistic EDFs. The LN method renders the values of calculated moments of inertia much closer to the data $[20,81]$ and at the same time much less sensitive to the underlying shell structure, at variance with the data, cf. the discussion in Ref. [21].

\section{Conclusions}

In this work we have used three different EDF approaches, each for two different parameter sets, so as to gain some insight into the degree of systematic uncertainties that are related to applying these approaches to spectroscopic properties of heavy nuclei at the gateway to superheavy region. On the one hand, we concluded that the overall coarse description of several 
spectroscopic properties of nuclei in this region is, in general, correct. On the other hand, we identified numerous smaller or larger differences between the results obtained within these different EDFs. In particular, none of the studied global EDF parameterisations precisely describes variations of the shell structure seen in experiment. This can be associated with small deficiencies of the obtained deformed shell properties.

On the scale of very precise spectroscopic experimental data, differences between various EDF approaches are large. They are of the same order as the degree of differences with experiment. This points to still fairly large systematic uncertainties inherent to the models currently in use. Moreover, none of the studied models could at present be identified as the one that systematically performs significantly better than another one. However, it is interesting that on the average the accuracy of the description of the excitation energies of deformed one-quasiparticle states in the nobelium region is substantially better than the accuracy of the description of the energies of dominant single-particle states at spherical shape in odd-mass nuclei neighbouring to doubly magic ${ }^{208} \mathrm{~Pb}$ (even as compared with the calculations which include particle-vibration coupling [13, 82].

The obtained results suggest that further work on improving the performance of the EDF methods is very much required. First, one can hope that within the existing forms of EDFs, one can still find better global parametrizations. For Skyrme EDFs, this route has already been explored and a negative conclusion was reached [27], but for covariant and Gogny EDFs similar work has not yet been performed. Second, one can hope that various beyond-mean-field corrections, not included in the present analysis, may have strong impact on the results, and thus modify the current conclusions. At least that happens in the covariant framework, where accounting for the (quasi)particle-vibration coupling improves substantially the accuracy of the description of predominantly single-particle states in spherical medium and heavy nuclei $[54,15]$. In addition, there is still a possibility of building new functionals, with beyond-mean-field effects incorporated from the very beginning. Again, for the Skyrme EDFs, employed together with odd-particle polarization effects included, a negative conclusion has recently been reached [82]. However, so far in the covariant and Gogny EDF approaches no such studies have been performed, and such route has to be explored. Third, one can also attempt building new classes of EDFs, where systematic expansions within different schemes are used. Only very recently this route was started to be explored in non-relativistic EDFs, see, 
Refs. $[83,84,85,86,87,88,89,90,91]$, so its eventual impact on the physics discussed here is not yet known.

\section{Acknowledgements}

This material is based upon work supported by the U.S. Department of Energy, Office of Science, Office of Nuclear Physics under Award Nos. DE-SC0013037 and DE-SC0008511 (NUCLEI SciDAC Collaboration), by the NNSA's Stewardship Science Academic Alliances Program under Award No. DOE-DE-NA0002574, by the Academy of Finland and University of Jyväskylä within the FIDIPRO program, by the Polish National Science Center under Contract No. 2012/07/B/ST2/03907, by the ERANET-NuPNET grant SARFEN of the Polish National Centre for Research and Development (NCBiR), by the CNRS/IN2P3 through PICS No. 6949, by the MINECO grants Nos. FPA2012-34694 and FIS2012-34479, and by the Consolider-Ingenio 2010 program MULTIDARK CSD2009-00064. We acknowledge the CSC-IT Center for Science Ltd., Finland, for the allocation of computational

resources. Computations were also performed using HPC resources from the MCIA (Mésocentre de Calcul Intensif Aquitain) of the Université de Bordeaux and of the Université de Pau et des Pays de l'Adour.

\section{References}

[1] R.-D. Herzberg and P.T. Greenlees, Prog. Part. Nucl. Phys. 61, 674 (2008).

[2] Ch. Theisen, P.T. Greenlees, T.-L. Khoo, P. Chowdhury, and T. Ishii, contribution to this volume.

[3] R.R. Chasman, I. Ahmad, A.M. Friedman and J.R. Erskine, Rev. Mod. Phys. 49, 833 (1977).

[4] J.L. Egido and L.M. Robledo, Phys. Rev. Lett. 85, 1198 (2000).

[5] A. Sobiczewski, I. Muntian, and Z. Patyk, Phys. Rev. C 63, 034306 (2001).

[6] T. Duguet, P. Bonche, and P.-H. Heenen, Nucl. Phys. A679, 427 (2001). 
[7] M. Bender, P. Bonche, T. Duguet, and P.-H. Heenen, Nucl. Phys. A723, 354 (2003).

[8] A.V. Afanasjev, T.L. Khoo, S. Frauendorf, G.A. Lalazissis, and I. Ahmad, Phys. Rev. C 67, 024309 (2003).

[9] D. Vretenar, A.V. Afanasjev, G.A. Lalazissis, and P. Ring, Phys. Rep. 409, 101 (2005).

[10] J.-P. Delaroche, M. Girod, H. Goutte, and J. Libert, Nucl. Phys. A771, 103 (2006).

[11] A. Sobiczewski and K. Pomorski, Prog. Part. Nucl. Phys. 58, 292 (2007).

[12] A. Sobiczewski, Radiochim. Acta 99, 395 (2011).

[13] A.V. Afanasjev and S. Shawaqfeh, Phys. Lett. B706, 177 (2011).

[14] Zhen-Hua Zhang, Xiao-Tao He, Jin-Yan Zeng, En-Guang Zhao, and Shan-Gui Zhou, Phys. Rev. C 85, 014324 (2012).

[15] E. Litvinova, Phys. Rev. C 85, 021303 (2012).

[16] H.L. Liu, F.R. Xu, and P.M. Walker, Phys. Rev. C 86, 011301 (2012).

[17] M. Warda and J.L. Egido, Phys. Rev. C 86, 014322 (2012).

[18] V. Prassa, T. Nikšić, G.A. Lalazissis, and D. Vretenar, Phys. Rev. C 86, 024317 (2012).

[19] Zhen-Hua Zhang, Jie Meng, En-Guang Zhao, and Shan-Gui Zhou, Phys. Rev. C 87, 054308 (2013).

[20] A.V. Afanasjev and O. Abdurazakov, Phys. Rev. C 88, 014320 (2013).

[21] Yue Shi, J. Dobaczewski, and P.T. Greenlees, Phys. Rev. C 89, 034309 (2014).

[22] J. Dobaczewski, W. Nazarewicz, and P.-G. Reinhard, J. Phys. G: Nucl. Part. Phys. 41074001 (2014).

[23] P.-G. Reinhard, M. Rufa, J. Maruhn, W. Greiner, and J. Friedrich, Z. Phys. A 323, 13 (1986). 
[24] G.A. Lalazissis, S. Karatzikos, R. Fossion, D. Pena Arteaga, A.V. Afanasjev, and P. Ring, Phys. Lett. B671, 36 (2009).

[25] M. Bender, P.-H. Heenen, and P.-G. Reinhard, Rev. Mod. Phys. 75, 121 (2003).

[26] E. Chabanat, P. Bonche, P. Haensel, J. Meyer, and R. Schaeffer, Nucl. Phys. A 635, 231 (1998); [Erratum, ibid. A 643, 441 (1998)].

[27] M. Kortelainen, J. McDonnell, W. Nazarewicz, E. Olsen, P.-G. Reinhard, J. Sarich, N. Schunck, S.M. Wild, D. Davesne, J. Erler, and A. Pastore, Phys. Rev. C 89, 054314 (2014).

[28] S. Péru and M. Martini, Eur. Phys. J. A 50, 88 (2014).

[29] J.-F. Berger, M. Girod, and D. Gogny, Comput. Phys. Comm. 63, 365 (1991).

[30] S. Goriely, S. Hilaire, M. Girod, and S. Péru, Phys. Rev. Lett. 102, 242501 (2009).

[31] P. Bonche, H. Flocard, and P.-H. Heenen, Nucl. Phys. A467, 115 (1987).

[32] B. Gall, P. Bonche, J. Dobaczewski, H. Flocard, and P.-H. Heenen, Z. Phys. A 348, 183 (1994).

[33] J. Terasaki, P.-H. Heenen, P. Bonche, J. Dobaczewski, and H. Flocard, Nucl. Phys. A593, 1 (1995).

[34] V. Hellemans, P.-H. Heenen and M. Bender, Phys. Rev. C 85014326 (2012).

[35] W. Ryssens, V. Hellemans, M. Bender, and P.-H. Heenen Comp. Phys. Comm. 187, 175 (2015).

[36] G. Bertsch, J. Dobaczewski, W. Nazarewicz and J. Pei, Phys. Rev. A 79, 043602 (2009).

[37] N. Schunck, J. Dobaczewski, J. McDonnell, J. Moré, W. Nazarewicz, J. Sarich, and M.V. Stoitsov, Phys. Rev. C 81, 024316 (2010).

[38] V. Hellemans, A. Pastore, T. Duguet, K. Bennaceur, D. Davesne, J. Meyer, M. Bender, and P.-H. Heenen, Phys. Rev. C 88064323 (2013). 
[39] T. Duguet, P. Bonche, and P.-H. Heenen, Nucl. Phys. A 679, 427 (2001).

[40] A. Chatillon, Ch. Theisen, P.T. Greenlees, G. Auger, J.E. Bastin, E. Bouchez, B. Bouriquet, J. M. Casandjian, R. Cee, E. Clément, R. Dayras G. de France, R. de Toureil, S. Eeckhaudt, A. Görgen, T. Grahn, S. Grévy, K. Hauschild, R.-D. Herzberg P.J.C. Ikin, G.D. Jones, P. Jones, R. Julin, S. Juutinen, H. Kettunen, A. Korichi, W. Korten, Y. Le Coz, M. Leino, A. Lopez-martens, S.M. Lukyanov, Yu.E. Penionzhkevich, J, Perkowski, A. Pritchard, P. Rahkila, M. Rejmund, J. Saren, C. Scholey, M.G. Saint-Laurent, C. Simenel, Yu.G. Sobolev, Ch. Stodel, J. Uusitalo, A. Villari, M. Bender, P. Bonche and P.-H. Heenen, Eur. Phys. J. A 30, 397-411 (2006).

[41] A. Chatillon, Ch. Theisen, E. Bouchez, P.A. Butler, E. Clément, O. Dorvaux, S. Eeckhaudt, B.J.P. Gall, A. Görgen, T. Grahn, P.T. Greenlees, R.-D. Herzberg, F. Hessberger, A. Hürstel, G.D. Jones, P. Jones, R. Julin, S. Juutinen, H. Kettunen, F. Khalfallah, W. Korten, Y. Le Coz, M. Leino, A.-P. Leppänen, P. Nieminen, J. Pakarinen, J. Perkowski, P. Rahkila, M. Rousseau, C. Scholey, J. Uusitalo, J.N. Wilson, P. Bonche, and P.-H. Heenen, Phys. Rev. Lett. 98, 132503 (2007).

[42] S. Ketelhut, P.T. Greenlees, D. Ackermann, S. Antalic, E. Clément, I.G. Darby, O. Dorvaux, A. Drouart, S. Eeckhaudt, B. J. P. Gall, A. Görgen, T. Grahn, C. Gray-Jones, K. Hauschild, R.-D. Herzberg, F. Heßberger, U. Jakobsson, G.D. Jones, P. Jones, R. Julin, S. Juutinen, T.-L. Khoo, W. Korten, M. Leino, A.P. Leppänen, J. Ljungvall, S. Moon, M. Nyman, A. Obertelli, J. Pakarinen, E. Parr, P. Papadakis, P. Peura, J. Piot, A. Pritchard, P. Rahkila, D. Rostron, P. Ruotsalainen, M. Sandzelius, J. Sarén, C. Scholey, J. Sorri, A. Steer, B. Sulignano, Ch. Theisen, J. Uusitalo, M. Venhart, M. Zielinska, M. Bender, and P.-H. Heenen, Phys. Rev. Lett. 102212501 (2009).

[43] M. Bender and P.-H. Heenen, J. Phys. Conf. Series 420, 012002 (2013).

[44] S. Ćwiok, W. Nazarewicz, and P.H. Heenen, Phys. Rev. Lett. 83, 1108 (1999).

[45] C. Rigollet, P. Bonche, H. Flocard, and P.-H. Heenen, Phys. Rev. C 59, 3120 (1999). 
[46] S. Ćwiok, P.-H. Heenen, and W. Nazarewicz, Nature 433, 709 (2005).

[47] N. Schunck, J. Dobaczewski, J. McDonnell, W. Satuła, J.A. Sheikh, A. Staszczak, M. Stoitsov, and P. Toivanen, Comput. Phys. Commun. 183, 166 (2012).

[48] M. Bender, J. Dobaczewski, J. Engel, and W. Nazarewicz, Phys. Rev. C65, 054322 (2002).

[49] L.M. Robledo, R.N. Bernard, and G.F. Bertsch, Phys. Rev. C 89, 021303(R) (2014).

[50] L.M. Robledo, R. Bernard, and G.F. Bertsch, Phys. Rev. C 86, 064313 (2012).

[51] L.M. Robledo and G.F. Bertsch, Phys. Rev. C 84, 014312 (2011).

[52] A.V. Afanasjev, P. Ring, and J.König, Nucl. Phys. A676, 196 (2000).

[53] S.E. Agbemava, A.V. Afanasjev, D. Ray, and P. Ring, Phys. Rev. C 89, $054320(2014)$.

[54] E.V. Litvinova and A.V. Afanasjev, Phys. Rev. C 84, 014305 (2011).

[55] A.V. Afanasjev and H. Abusara, Phys. Rev. C81, 014309 (2010).

[56] A.V. Afanasjev and H. Abusara, Phys. Rev. C 82, 034329 (2010).

[57] W. Koepf and P. Ring, Nucl. Phys. A493, 61 (1989).

[58] P. Ring, R. Beck, and H.J. Mang, Z. Phys. 231, 10 (1970).

[59] J.L. Egido, H.J. Mang, and P. Ring, Nucl. Phys. A334, 1 (1980).

[60] P. Ring and P. Schuck, The Nuclear Many-Body Problem (SpringerVerlag, Berlin, 1980).

[61] S. Raman, C.W. Nestor, and P. Tikkanen, At. Data Nucl. Data Tables 78, 1 (2001).

[62] K. Rutz, M. Bender, T. Bürvenich, T. Schilling, P.-G. Reinhard, J.A. Maruhn, and W. Greiner, Phys. Rev. C 56, 238 (1997). 
[63] M. Bender, K. Rutz, P.-G. Reinhard, J.A. Maruhn, and W. Greiner, Phys. Rev. C60, 034304 (1999).

[64] Y. Shi, D.E. Ward, B.G. Carlsson, J. Dobaczewski, W. Nazarewicz, I. Ragnarsson, and D. Rudolph, Phys. Rev. C 90, 014308 (2014).

[65] T. Lesinski, M. Bender, K. Bennaceur, T. Duguet, and J. Meyer, Phys. Rev. C 76, 014312 (2007).

[66] M. Bender, K. Bennaceur, T. Duguet, P.-H. Heenen, T. Lesinski, and J. Meyer, Phys. Rev. C 80, 064302 (2009).

[67] Evaluated Nuclear Structure Data File (ENSDF) located at the website (http://www.nndc.bnl.gov/ensdf/) of Brookhaven National Laboratory. ENSDF is based on the publications presented in Nuclear Data Sheets (NDS) which is a standard for evaluated nuclear data. Adopted experimental one-quasiparticle levels are taken from the NDS articles published during 2001-2014.

[68] A.K. Jain, R.K. Sheline, P.C. Sood, and K. Jain, Rev. Mod. Phys. 62, 393 (1990).

[69] K. Hauschild, M. Rejmund, H. Grawe, E. Caurier, F. Nowacki, F. Becker, Y. Le Coz, W. Korten,J. Döring, M. Górska, K. Schmidt, O. Dorvaux, K. Helariutta, P. Jones, R. Julin, S. Juutinen, H. Kettunen, M. Leino, M. Muikku, P. Nieminen, P. Rahkila, J. Uusitalo, F. Azaiez, and M. Belleguic, Phys. Rev. Lett. 87, 072501 (2001).

[70] A. P. Leppänen, J. Uusitalo, M. Leino, S. Eeckhaudt, T. Grahn, P. T. Greenlees, P. Jones, R. Julin, S. Juutinen, H. Kettunen, P. Kuusiniemi, P. Nieminen, J. Pakarinen, P. Rahkila, C. Scholey, and G. Sletten, Phys. Rev. C 75, 054307 (2007).

[71] L. Bonneau, P. Quentin, and P. Möller, Phys. Rev. C 76, 024320 (2007).

[72] R. Rodriguez-Guzman, P. Sarriguren, and L.M. Robledo, Phys. Rev. C 82, 061302(R) (2010).

[73] R. Rodriguez-Guzman, P. Sarriguren, and L.M. Robledo, Phys. Rev. C 83044307 (2011). 
[74] W. Satuła, J. Dobaczewski, and W. Nazarewicz, Phys. Rev. Lett. 81, 3599 (1998).

[75] G. Audi, F.G. Kondev, M. Wang, B. Pfeiffer, X. Sun, J. Blachot, and M.MacCormick, Chinese Physics C36, 1157 (2012); G. Audi, M. Wang, A.H. Wapstra, F.G. Kondev, M. MacCormick, X. Xu, and B. Pfeiffer, Chinese Physics C36, 1287 (2012); M. Wang, G. Audi, A.H. Wapstra, F.G. Kondev, M. MacCormick, X. Xu, and B. Pfeiffer, Chinese Physics C36, 1603 (2012).

[76] J.J. Li, W.H. Long, J. Margueron, and N. Van Giai, Phys. Lett. 732, 169 (2014).

[77] T. Bürvenich, K. Rutz, M. Bender, P.-G. Reinhard, J.A. Maruhn, and W. Greiner, Eur. Phys. J. A3, 139 (1998).

[78] P. T. Greenlees, J. Rubert, J. Piot, B.J.P. Gall, L.L. Andersson, M. Asai, Z. Asfari, D.M. Cox, F. Dechery, O. Dorvaux, T. Grahn, K. Hauschild, G. Henning, A. Herzan, R.-D. Herzberg, F.P. Heßberger, U. Jakobsson, P. Jones, R. Julin, S. Juutinen, S. Ketelhut, T.-L. Khoo, M. Leino, J. Ljungvall, A. Lopez-Martens, R. Lozeva, P. Nieminen, J. Pakarinen, P. Papadakis, E. Parr, P. Peura, P. Rahkila, S. Rinta-Antila, P. Ruotsalainen, M. Sandzelius, J. Sarén, C. Scholey, D. Seweryniak, J. Sorri, B. Sulignano, Ch. Theisen, J. Uusitalo, and M. Venhart, Phys. Rev. Lett. 109, 012501 (2012).

[79] M. Asai, private communication.

[80] A.V. Afanasjev, J. König, P. Ring, L.M. Robledo, and J.L. Egido, Phys. Rev. C 62, 054306 (2000).

[81] A.V. Afanasjev, Phys. Scr. 89, 054001 (2014).

[82] D. Tarpanov, J. Dobaczewski, J. Toivanen, and B.G. Carlsson, Phys. Rev. Lett. 113, 252501 (2014).

[83] B.G. Carlsson and J. Dobaczewski, Phys. Rev. Lett. 105, 122501 (2010).

[84] M. Stoitsov, M. Kortelainen, S.K. Bogner, T. Duguet, R.J. Furnstahl, B. Gebremariam, and N. Schunck, Phys. Rev. C 82, 054307 (2010). 
[85] B. Gebremariam, S.K. Bogner, and T. Duguet, Nucl. Phys. A 851, 17 (2011).

[86] J. Dobaczewski, K. Bennaceur, and F. Raimondi, J. Phys. G: Nucl. Part. Phys. 39, 125103 (2012).

[87] J. Sadoudi, T. Duguet, J. Meyer, and M. Bender, Phys. Rev. C 88, 064326 (2013).

[88] K. Bennaceur, J. Dobaczewski, and F. Raimondi, EPJ Web of Conf. 66, 02031 (2014).

[89] F. Raimondi, K. Bennaceur, and J. Dobaczewski, J. Phys. G: Nucl. Part. Phys. 41, 055112 (2014).

[90] T. Duguet, J. Phys. G: Nucl. Part. Phys. 42, 025107 (2015).

[91] T. Duguet, M. Bender, J.-P. Ebran, T. Lesinski, and V. Somà, arXiv:1502.03672. 